\title{
LA RADIOATTIVITA' DELLA TERRA
}

\author{
C. Festa - M. Sivtangelo
}

\section{I - Metorli di misura}

1. - La individuazione delle sostanze radioallive nella crosta terrestre ha una grande importanza dal punto di visla gcofisico, in quanto la loro presenzal ò molto verosimilmente comnessa con le diverse manifestazioni del nostro pianeta ed e legata alla sua storia passatta e futura.

le misure in silu della radioallivila di materiali rhe rotituiscono la tera sono necesariamente limitate a profondia relativamente piecole: le ricerehe più recenti sono state epinte nei sondaggi pelroliferi a circa 3000 m (1). Ogni supposizione sulla distribuzione delle sostanze radioatlive in tutla la erra fondala sulla estrapolazione dei risultati sperimentali is in verita alquanto arbilraria.

Quantuntre siano state effelluale misure anche su materiali provenienti da profondià comiderevoli (lave, aceque profonde, gas), il numero di tali delerminazioni a ancou troppo scarso perehé si po-sano formulare delle ipole-i allendibili.

Molte vaste regioni della terra non sono slate ancora esplorate sotto questo aspello. A questo proposito è augurabile che fra non molı anni possa aversi una carta radioggcolog̣ica complela e aggiornatal $\left({ }^{2}\right)$.

Se si limitano le nostre considerazioni allo strato più superfieiale, biogna rilerare subito due fenomeni: il contenuto in sostanze radioaltive deceace man mano ehe i procede dagli sitati piu superfieciali a ruelli più profendi. mentre al contrario la densità media delle masse continentali io minore di quella del basalto sub-continenlale e degli strali ancor paì prolondi. Il tenore radioallivo delle mas. se continentali e sub-continentali s riassunto nella labella $I\left(^{3}\right)$ che ha un catrattere puramente orientativo: 


\section{TABELLA I}

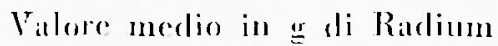

$$
\begin{aligned}
& \text { per gr di so-lanzat } \\
& 3.4 .10^{-1-} \\
& 11,75 .\left[11^{-1-2}\right. \\
& 1,1.9 .10^{1:} \\
& 0.33 .10^{\mathrm{J}} \\
& 11,30,10^{-1:}
\end{aligned}
$$

$$
\begin{aligned}
& \text { Maree rontinentali } \\
& \text { Basalto -ub-eontinentale } \\
& \text { ) sub-oceanico } \\
& \text { Dunite e analonhi } \\
& \text { ficlowite }
\end{aligned}
$$

Nei minerali radionulivi si ritrovano tulli gli elementi delle tre famiglie in equilibrio radioallivo (v. app). Ill): quelli il rui periodo $\therefore$ mole breve sono presenti in quantia infentesime. Riportiamo la seguente labella da Joliot-Curie (') dalla equale possono rilevarsi le quantita relatioe dei radiocementi presenti nei minerali di lranio r. (li Torio.

TIBELLA II

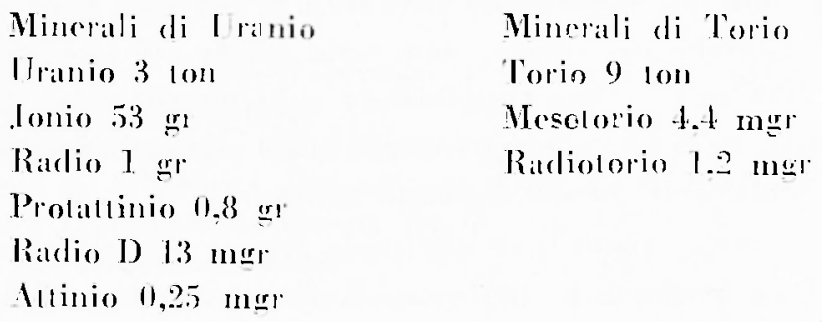

I minerali sli limanio o di loria sono: wli o-sidi (Perhblenda. Dorianite), i prodotti di alterazione degli onidi (Curibe. Bectuerellite. Soddite. Troile) i fosfati (Autumite, Calcolite, Monaxite), i vinatali (Camotili) i niobali e lanbalali (Samarkile, Belalile). Jal Pechblenda i il minerale principale di fluthamento per l'estraxione dell'tranio a do swoi derivali: asa si trova principalmente in

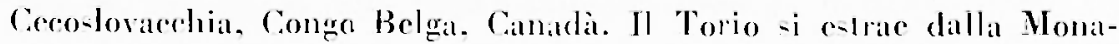
zite (fosfato di Torio e tere rare) proveniente dal Brasile. Stati Tnili, India ece. Oltre a questi minerali vengono lavorati per l'estazione allotranio, Radio, 'Torio e Terre rare: l'Aummile nel Portogallo, la Carnotile, la Bebatile, minerale alblondante nelloi-ola del Marlatgavear e nel griacimento wrinifero nel Kalangal.

Gencralmente fuesti minerali sono intimamente mescolati alle roree a ai tereni oppure sono diseminali solto forma di piccoli 


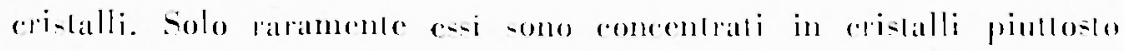
voluminosi o in mane compalle nei filoni ronero-i. Tulli i minerali di Dranio a di Torio ronlengono Piombo ed Elio: il primo ir il prodollo finale delle tratormazioni radioallive mentre il seondo pro-

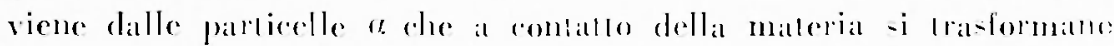
in Elio ordinario.

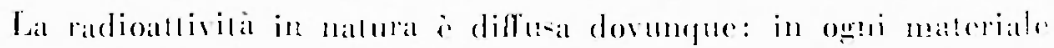

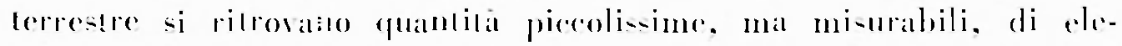
menti radioallivi. Anche gli organimi visenti concentrano elementi a periodo hreve (Radio. Mesolorio) ollere a rentenere i! Polacio ra-

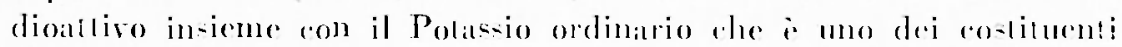
principali del mondo hiologico.

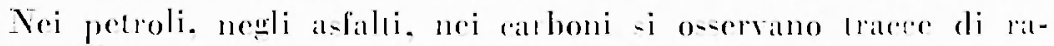
dioallivila e di Elio provenienle da di-inlegrazioni. Molls aldue infine sono ricrle di Raton e di ala elementi radioallivi. Parlino latmente interesinti sono le riecrelle -ul conlenulo in elementi ra-

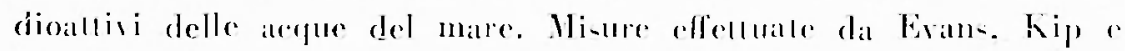

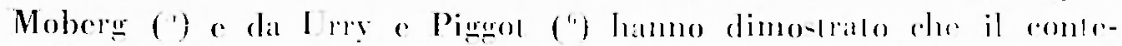

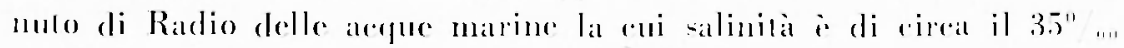

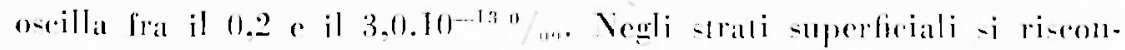
trano i valori pio ha-ci mentre il valore mas-imo corrisponde a misure effelluale sll alefue a romballo coa i sedimenli matrini.

Il riconosianento ed il do-aggio delle so-lanze radioallire di-

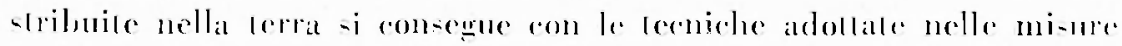

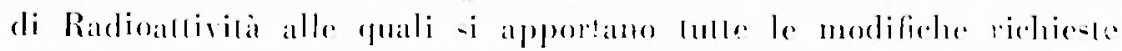
dalla particolare natura delle riecrele geofi-ieloe.

2.-- I melodi per la rivelazione o lo - fudio quantiativo delle so-tange radioallive -ia malurali che arlificiali sono basalli sulle pare

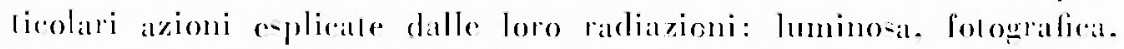
ionizzante eece Fai sono: 1) i! motolo delle scintillazioni: 2) la ra-

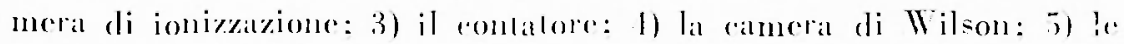
emulsioni sensibili.

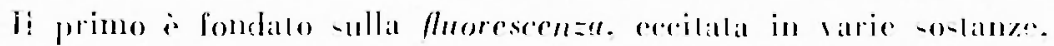
romer il plalinorianuro di Bario. il -olfuro di Zineo ene dalle radia. zioni eme-se dignti elementi radioallisi. In parlieolare quando -ullo

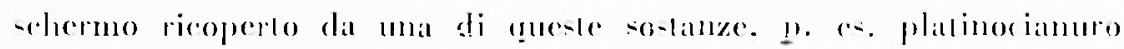

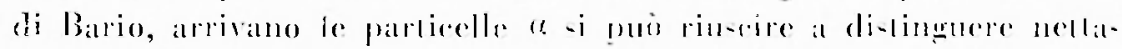
mente l'effello individuale di ciasenma parlieclla -ollo forma di as sinlillazione n. Gerolre pero atoperare mat soregente non lroppo allisa 
ed osemalre il fenomeno con un opportuno ingrandimento. In lale "a-o un osecratore abitualo puo contare il 90-95\% delle particelle im.identi. Questo semplicissimo melodo ha reso serrigi incslimabili melle prime recrehe di radioaltivita. Con esso Rublerford (') hat rffeltuato le clasiche esperienze sula diffusione delle particelle u

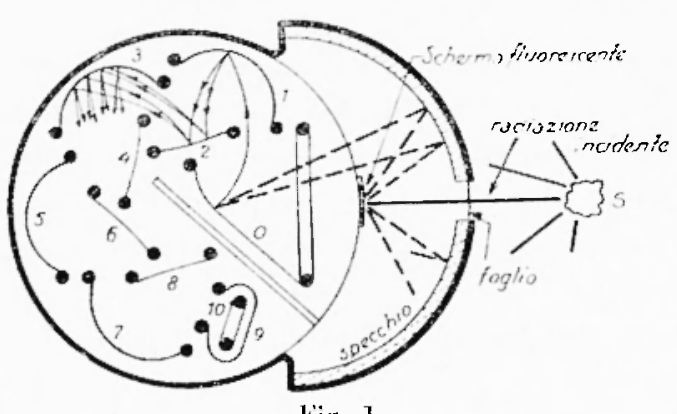

l:ig. 1

0 : fotocatado; 1-9: clellodi ad emissione secondaria: 10: anodo r, insicme ron Chadwiok. ha scoperto la radioatlivilà arlificiale. Ja sen-ibilisa di fuesto metodo puó esere aumentala di parecelui ordini di grandezza sostituendo all'occhio un fotomoltiplicatore clellronico, munilo di im opportumo si=lema ollico e di nuno schermo fluorencente. Si oltiene in lal modo un mivelalore $\left(^{s}\right)$ di radiazioni di qualsiasi tipo (particelle e e P, ragri $x$ e y eer.). In fig. I riportiamo la co-truzione dellagliala di queso strumento. Ia radiazione (p. en. una parlicella a) entra lalla funeslia e poduce una scintillazione sullo -rhermo fluoresente. Lo specrho foralizza sull'area sem-ibile del fototatodo la luce emessa dallo schermo fluorescente. Ciaseun fotocleltene, aceclerato dalla differenza di polenziale di un rentinaio di volts -sictenli fra il calode e il primo clettrodo ad emisione secondaria (r. anche fig. 2), cstrace da fues tultimo quatlro o cincue elellroni, i fuali a loio volta rengono sospinti sul secondo elettrodo e cosi via.

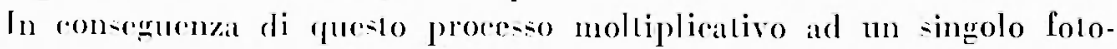
elenrone corrisponde alleuseila una valan-

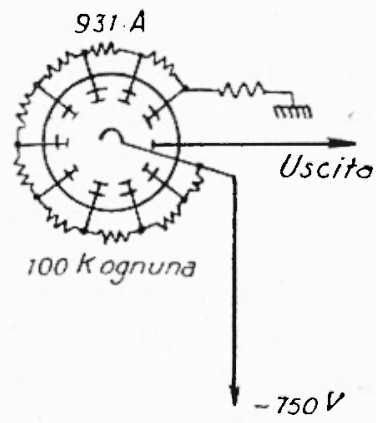

Fig. 2

tirruito del folomoltiplicatore gua di rirca $10^{6}$ eletroni.

Questo strumento semplice e poco di-pendiono puo essere usilo per un ampio intervallo di intentita ed ha un rendimenlo elic, per le particelle $u$ e $\beta$, si aggira con tulla probabilita attorno al $100 \%$.

Poiché il sccondo e il ter\%o melodo -ono molto wati nelle ricerele di Gcofisica ci proponiamo di parlarne piu diflnsamente in seguilo, limilandoci per ora a segnalare il fallo rlie ambedue i me- 
lodi sfrutano l'azione ionizante delle radiazioni emesie dalle =oslanze radioallive.

Passiamo quindi a dare un remno del quarto melodo. noto romunemente con il nome di melodo della camera di Wilion (?). Esso e basalo sula condensizione del vapor d'acefua soprasaluro atlorno agli ioni rlye le parlicelle, emese dalle sostanze radioallive, senerano lungo il loro pereorso.

La cancra di Wilson è composa schemalicamente da un cilinslro incelalico hi (r. fig. 3) nel quale può scorrere un pistone $i$. Sul rijindio $H$ viene sovrapposto un altro cilindro di vetro $T$ dello slesso dianetro, chima superiojmente da ma lastra di vetro $t$.

Il gat all'interno del rilindlo è situro di vapor d'actua. Ein rapido movimento del pistone produce ma copansione adiabialiar del

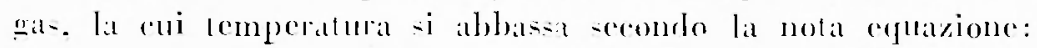

$$
T v^{\prime-}=\cos
$$

con $\gamma=c_{p} / c_{v}$. Comseguentemente il rapore all"interno della camera diviene soprasaluro. Oral ie noto dhe un vapore soprasaturo lende a formare la nehbia onia a condensarsi in goceioline. Se la soprazatuazione non is lroppo forte la condensazione si rerifica solo in presonza di centri di condensazione, yuali i grani di pulviscolo atmosferiro o gi ioni. Perciò se il gars rontemuta allintrmo della camera econte da anal sia pull minima trateria di pulvicoolo almoserico, il rapore acture si rondensa solo sugli ioni formali lungo il loro pereorso dalle particelle cariehe (partieclle a, elcllroni negallivi e posilivi eac.) e-

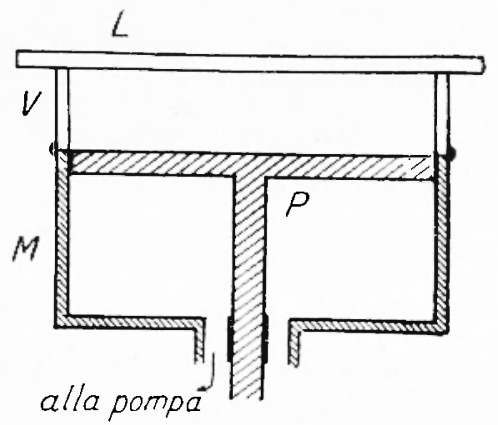

Fig. 3 mese dal preparato radioattivo. Disponendo ma inlental sorẹcente di luce di lato e nua marehina folografira di soplat, s powono forlografare le labictlorie delle ingole particelle, le quali sono traceiate da un soltile filo di nelibia.

Come risulta dalle fotografie (fig. 1 e j). le tracee delle parlirelie a si distinguono a prima vista da quelle delle particelle $\beta$, in quanto, a dausa del diverso numero di ioni per em (v. appendice II), labpetto delle prime differise considerevolmente da quello delle seconde. Mentre le lraree delle partirelle a sono dense e di spessore 
milorme, yuelle desli elellroni sono più sotlili e presentimo addensamenti irregolari di goredoline.

Dallat folografia di fige. 4 ri-ulta evidente la pre-enzal di due gruppi ben definiti di particelle ucon un pereoro nellamenle diflerente:

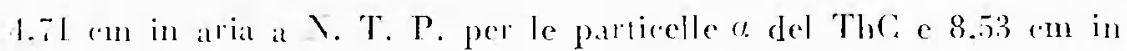
aria a J. T. P. per quello del The'.

In fig. 5a areanto alla trateria retlilinea e sotlile di una parliacila j. -ono evidenti le trane brevi. grose e ineurale degli eletroni Irnli. serondali di ragi X.

In pratica oceorrono aleme precatuzioni per il buon funziona-
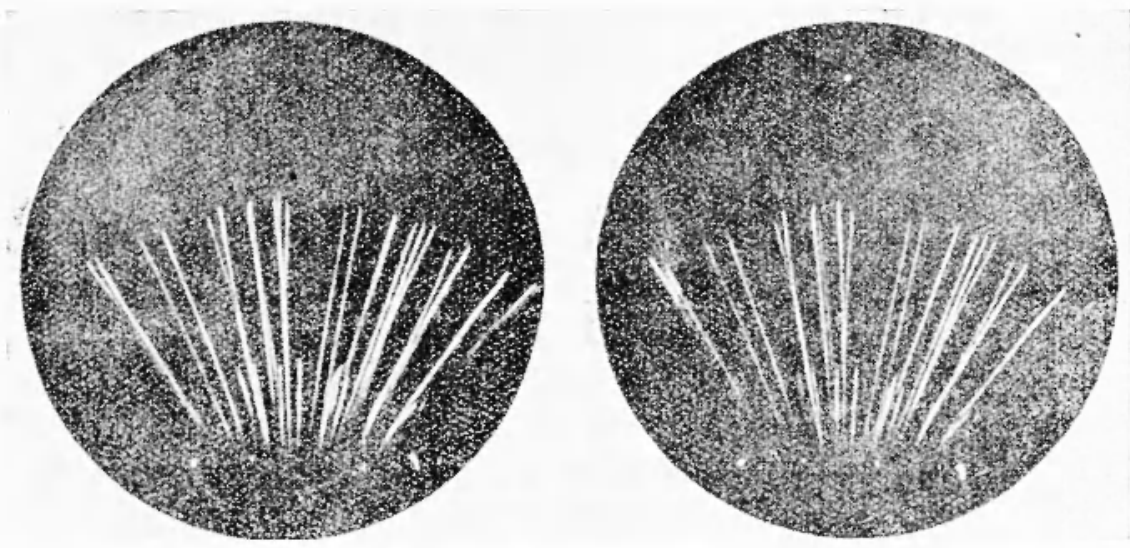

Fig. 4

mento dellapparee hio. Ad es per ollenere latere ben definile ace eorere ronferibe un valone ben delerminalo al "rapporlo di espan-ione" onia al rifperero fra il rolume iniziale e il volume finale oreupato dallat mi-relat gas-rapore. Queste valore dipende dal tipo di miscelat prat-vapore contenulo nella rancrat. Gli joni rhe si formano spontancamente a calloal della cosidelta "ionizzatione spontanca " della quale arremo oerasione di parlate in segulo. vammo eliminati in manielar da impedire rle agiseano come rentri di condensizione, producendo una nehlia diffu-al. Tale eliminaxione -i olliene modiante lat applicizione di un opportmo ampo elellico dhe vicne soppresso al momento dellat espantione.

se camele di Wileom possono esepe amomathe o no. Nel primo cino le expan-ioni -i -uredono a intersalli regolari. Un opportuno 
meceanimo manova il pistone, inseri-ce e loglie il rampo deltriero ece. Quando non i nece-inio oltenere un gran numero di fotorratie a la camera hat dimen-ioni con-iderevoli. dimodoche non sopportal espansioni troppo frequenti, conviene rinunziare al funzioramento aulomatico.

Con il metodo della camera ai $l l$ ilson si puó delermiHate anche lat ionizazione specitica delle particelle osia il mumero di ioni whe queste produrono per erm di percor=o. Infalti con opportuni areorgimenti is posibile realizzare foiografie nelle guali si riene a fontare il numero detle woccioline " ruindi di ioni per em di traceial. Se poi la camera di Wilson a tronat in un campo mitzneliec uniforme di inten-ilit $1 \mathrm{t}$. arente direzione paral-

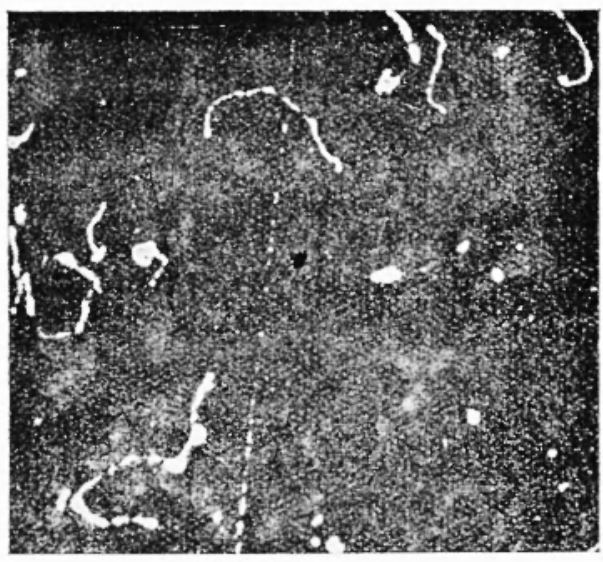

Fig. 5

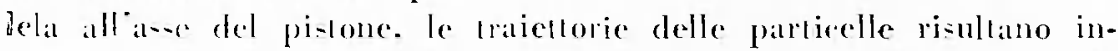
curralte in un reres o ace repo oppesto al serondat del semo della

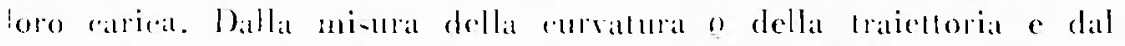
:alore del campo - i pieara limipuloo me dellat particellat mediante la formulat:

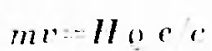

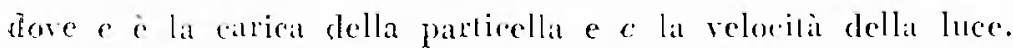

Il matewiore inconveniente presentalo dallat camera di $\mathbb{K}$ ileon ron-iste nel fatto whe tra una espantione e labltra occorre lasciare pariare un ecrto tempo per permetlere allat mi-eela gat-vapore di lomare in equilibrio termico con lambiente.

Lubimo metodo è quello delle emulsioni folografiche $\left({ }^{10}\right)$. Le particelle emesse dalle so-lanze madioaltive agi-cono sulla emulsione folografiea in maniera analoga a quella dei fotoni. producendo un ianerimento approwimalivamente proporzionale al potere ionizzante dellia particella. Le emulsioni semibili adoperate nelle ricerche di radioalliviti contengono unat quantití di alogemuro di Argento alIneno dieri volte matrione di quelle delle emul-ioni ordinarie. Le dimen-ioni dei ingroli granuli di alogenuro di Arento vartiano nei dif- 


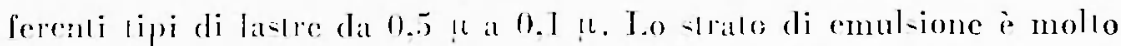
più-pen-s -- areal $\mathrm{l} / 10 \mathrm{~mm}$ - di quelo delle lastre ordinarie.

la considerevole densila degli alomi che roslitui-cono la emulsione folografue fa si rhe in essal jl pereorso delle particelle u a riducia a 1 loon del pereoro delle sese particelle in aria cosieché esse sono romplelamente assorbile dialla emul-ione folografieal. In una lastra eposta allat radiazione e risullano siluppali solo i gramuli fli alogenuro di Areneno colpili dalle particelle a lungo il loro pereoroo. Conseguentemente all'esime microseopiro le trabere si presentano rome lines costiluite da una sucessione di gramnli neri (v. fiğ. 6 e 7).

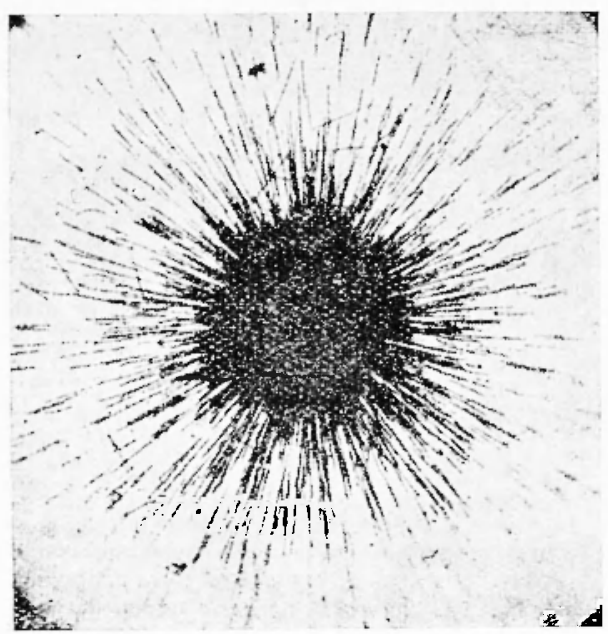

lị. 6

In lig. 6 le llatrece -lale prodolle da guantita di Radio invisibili al microreppio. La folografla es stalal ollenula seuotendo al disoprar della lat-

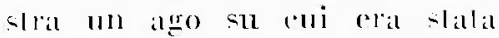
depo-ilala unal piecola quantita di Radio, in manicra da fir radere i frammenti di Radio - mlla la-lar. Ouando, dopo pochi giorni la lastra os slala sriluppatis. al appareal ropertal da numerose selle del lipo di quelle rappresentale in que- ta figurar. Le marese delle parlieclie $u$-i imidiano kla ma piecola areal siluala al rentro del mineroseopio.

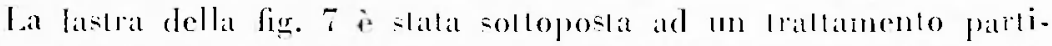
solare che ha permeso di incorporare la so-tamza radionaliva nellat amulsione folografieal. Ogni stellat clata prodotla dal deradimento di un nurleo di Radiolorio. Si notino aleranto allat slella rentrate

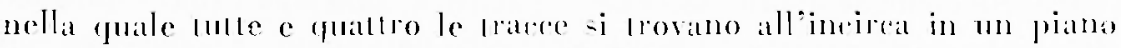

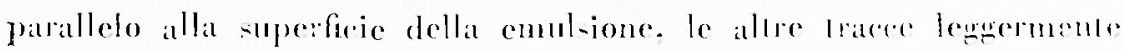
sfuocalc.

Evidentemente questo melodo ha molti junti di rontallo ron quello della ramera di Wilan. Ial lastra folografica offe il rantaggio di essere sempre senubile oltreche particolarmente sempliec ad u-are. 
Mollo spess le lrace sono visibili mediunle m microsenpio ordina!ro. Peó̀ le migrlori mirrofologratie si ollengrono con un obientivo ad immersione di grande aperlura il quale consente lesime dellagrliato della slutuma delle lracese. Quesi ohietlivi lamno una pro-

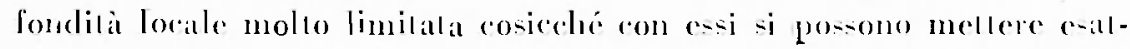
lamente a fuoco olo $\mathrm{i}$ erranuli della lraceia rontenuti in uno sratto molos soltile. In queste condizioni la folografia ing̣andila della lrandia non -i può ollenere ron tanat kolat po-il a meno rhe la traceia nom -ial interamente compreas in un piano pitrallelo alla lastrat. Altrimenti

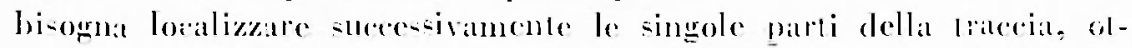
renendo una suecesione di fologerefe con le quali si puo realizzare un mosaico del lipo di fuelli adoperati nella aerofotog̣rammetria.

Con queso sistema si riese a rappresentare nel piano un fenomeno rlue arvene nello -pazio. Lo sesso metodo viene adollato nel ca-o in eni log̣gello a troppo grande per esere folografialo al microscopio con mal singola posa.

D) allro ('illlo non si puó fire a neno di nolare ale lo sviluppo alluale di queslo nuccodo con-ente la regi-labione folografiea delle sole parlicelle preanti. la rui ionizztzione specificis is rom-iderevole. $E$;

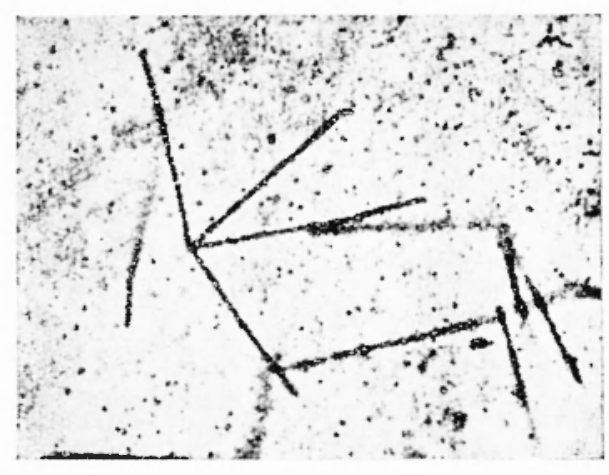

Fig. 7 reconlisima la nolizia della preparazione di emulaioni con le quali si sarchbe ollemula la folografla delle lrame di cleteroni lemli ("i).

Lo sviluppo e il fissaggio delle lawe riehiedono particolari precauzioni a causa del considerevole spesore delle emulsioni e della rilesante concentrazione di molecole di alogenuro di Ağ. Fino a poco rempo fa il contributo portalo da queso melodo alle riere che sulla radioallivili era relativamente searo in confronlo a quello del melodo di Wilson e dei melodi elellriei (ramera di ionizzazione e contalore), ma allualmente esso va alequi-tando un consderevole sviluppo dovulo alla sua e-lrema semplicita e alle imporlanti-ime seoperle che con esso sono slate confeguile da un ammo a que-la parte. In particolare esso consente di effelluare ricerelue sulla 


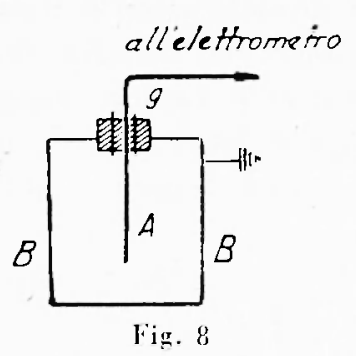

ratdioattivita natulale con una attrezzaluas realizathile in qual-iasi mode-to laboratorio.

3. - I costituenti esenziali di ma cumera di ionizzazione sono un reripiente mè. tallico e un elettrodo sostenuto da un supporto isslante ( $r$ fig. 8). Lat naturat e la prewione del gas contenuto all“interno della ramera variano a secondal dello spopo rhe -i propone la ricerea. Fra il recipiente e l'eletrodo si slabilisce ma differenzal di potenziale di qualche rentinaio di volt in maniera the l'azione ionizzante delle ostanze radioattive puó essere rivelala da

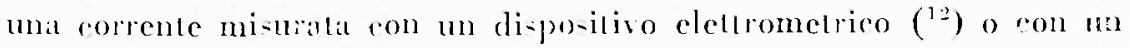

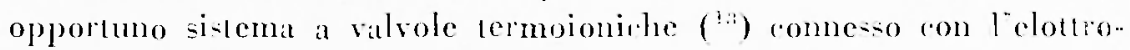
do centrale. Invere di un solo elettrodo entrale la camera puo ron. tenerne due, fra $i$ quali $-i$, slabili-ue la differenza di potenziale -ust. della, mentre il recipionte esterno, sollegato ron il suolo. sorve da schermo eletrostallico (r. fig. 9).
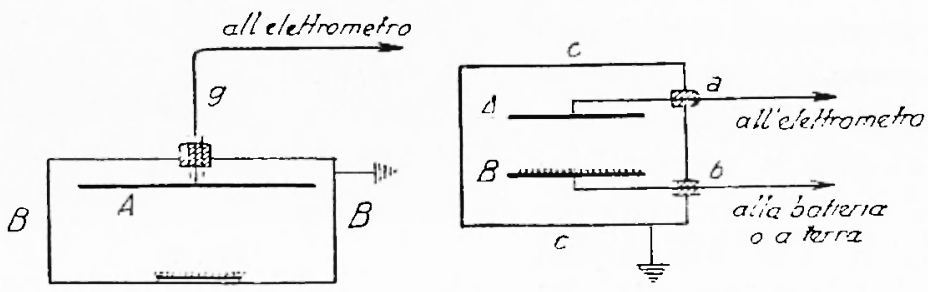

Fig. 9

Anche la forma dellat ranera e la di-posizione degli olellrodi dipendono dagli scopi che i propone l'esperienza.

Se $m$ e la quantila di un elemento radioattivo esperat in gr ad 4 il sto peso allomico, il numero degli alomi che si thatormano nelfunila di lempo is:

$$
\lambda m N / 4
$$

dowe i i la costante di disintegrizione dellelemento -teso ed $V$ il numero di Arogadro. Eridentemente que-io numero dere esiere nyuar le al numero di particelle emese dallelernento nell'uniti di tompo.

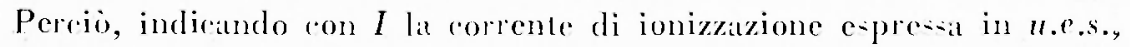
-i hat in definiliva:

$$
I-\operatorname{monel} 1
$$


dore $n$ è il numero di ioni prodolli da una singola particella ed e la carica cletlriea elementare.

La quantita $m$ di un elemento radioallivo rontenulo in man resta sostanza può essere dedotla dalla corrente di ionizzazione I. In prat. tica per poler applicare gluesta formula ron suffiente esallezzal orcorre rhe siano soldisfalle le secruenti rondizioni: 1) il pereoro delle particelle enese deve esauries nella ramela; 2) lat-sorbimento delle parlicelle da parle della sostmza the le emelle deve enere ridollo al minimo; 3) la corrente deve aver ragrgimlo il valore di "salualzione », ossia il valore per il quale lulli grli ioni generali nella ramerat raggriungono erli elellrodi senza arer morlo di ricombinar-i.

11 melodo più frequentemente adollato per la valulazione dellat radigallivila dei minerali di Radio consisle nell inflodure la so-linza in esame nella camera di ionizzazione e mi-urate il valore della rorreste di saturazione, la fuale é dovula quasi eselu-ivimenle al-

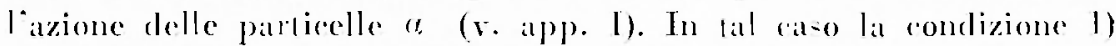
$\therefore$ senzaltro soddislalla (*). Però l'elevalo potere ionizanle delle palllieelle a la si che e.se vengano in parte asiorbile nellat =le-al so-lanzat che le emelle sirelhé quesle misure pur pre-enlando una ronsiderevole ulilita pratiea, non consentono ma grande presisione. A parilà di soslanza la correnle di salurazione può variare considerevolmente a secondal elue la superfueie su cui la sostanza viene distri. buila è più o meno escat. Per ollenere ma maggiore preci-ione ocrorre dunque disporre le sostanze radioatlive su frali abbalanzal sol-

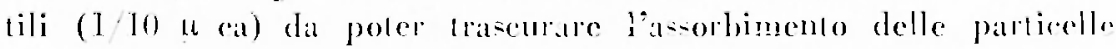

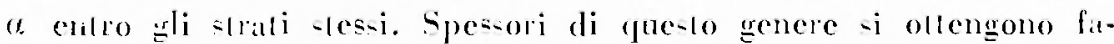
rilmeile nel raso dei co-liluenti dei deposili allivi o del Polonio. In aluri rasi (composti dell'tranio e del Radio) conviene ricorreres alla eviporazione di ma soluzione oppure alla sedimentazione di una sospensione molo fine in un liquido (p. es. eloroformio). Jal sosimza cosi funemente polverizzala e di-lribuila sll ma superfecie il piu possibile uniforme viene jntrodotla nella camera di ionizzazione mediante un conveniente supporlo (fur. 9). Le dimensioni della camera devono esere lali da ronanlire alle patticelle a di terminare il loro pereorso entro il gras in esia rontenulo. Lal rolrenle di salurazione $i$ che si misura in queste condixioni as-ume un signifualo ben previso: essa rappresental la meta della corrente di ionizzazione $I$ ri-ultanle

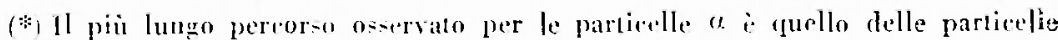
a det ThC': 8.63 'me a N.T.P. 


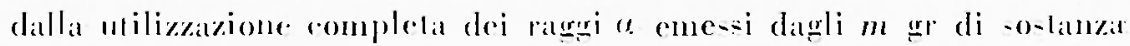
reaminala. L'altra melà viene assorhital dalla sostanzal slessal. Convirene generalmente confrontare la corrente $i$ minurata nella manterat sudelelta ron quella prodolla da un "campione" di ossido di Uranio

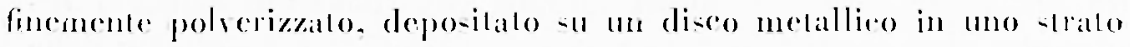
miforme e compatlo.

$\mathrm{E}$ ' wio rhe la condizione 3 ) è lanto meno odelisfalta. quanto

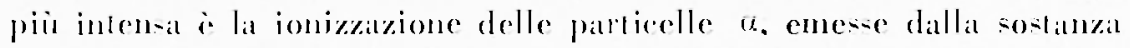
in enane. Si ricorre in lal at-o a parlicolari aceorwinenti sperimen-

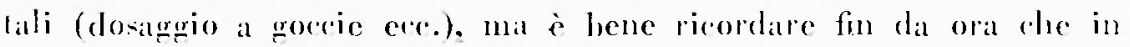
que-li casi la misura della corrente di ionizzazione puo enere va-

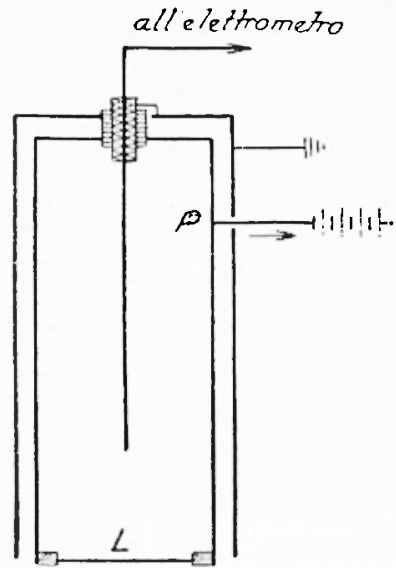

Fig. 10 lidamente so-tiluilat ron la numerazione direla delle particelle. Di que-lo metodu ci oceuperemo nel prosimo parabrafo.

Per l'eane delle radiazioni $\beta^{-1}$ adoperal mal anmeral di ionizatzione del lipo di lig. I0 nella quale la ionizgarome i.

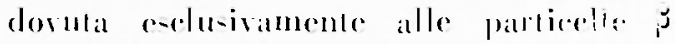
emesse dalla solegente ele penetrano ne!la camera allraverso la finestra $l$, ehilloal da

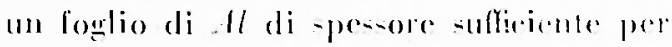
atrestare la erentuale radiazione a. In ge

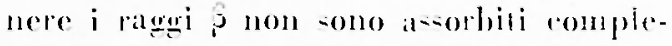
latmente dal gal della rameral. -inels in que-10 caso la rondizione l) non is sonddisfalla senzaltro e la corrente di sallowazione verà a dipendere dalle dimensioni della camera e dalla po-izione della sorgente rispello a questa. Th ogni ra-o sara bene alue ke dimenioni della camera simo magrgori di quelle delle camere pes

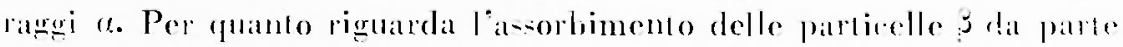
dellit -ostanza dalla quale sono emene vanno ripetule lute le ri-erve

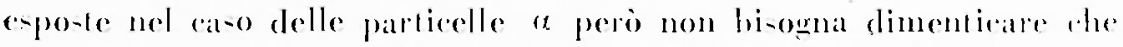
grli errori provenienti dall'a-sorbimento sono anto più pidolli quanta piì elevalo $\dot{a}$ il polere di penelrazione delle parlicelle $\beta$ osiervale.

Finora abhiano vito rhe le ramere di ionizzazione con-entoim lo studio qualitation e quantitation dei minerali radioallivi sia nel caso di comi-ione re come in quello di emi-wone $j$. Ma amele la radiazione ${ }^{\prime}$ ehe aceompagna ora una ora l'altra di queste radiazioni può esiere adoperalat a questo seopo quantunque la sua debole axione: 
ionizzante renda necesari particolari arrorengmenti: ramere di dimen-ioni piullosto grandi, riempimento con tras particolarmente assorbenti (per es. CIII), presioni superiori alla normale nellinterno della cameras.

D'alla parle la con-iderevole penetrazione dei rageri $\ddot{q}$ con-ente il dosingrio di sostanze contenute in ampolle saldale alla fiammia. Di qui la parlieolare sempliata di gue-lo melodo rhe non rirhicele mat-

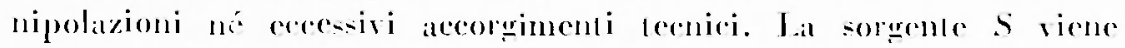
po-lil - 11 uno s-hermo di piombo di-po-lo -ulla parete -uperiore di mal ramera a grande diamedro $(20-30$ r.m) rome in fig. 11. I flambilalivi di Radio conlemuli in due ampolle, -imili e -imil-

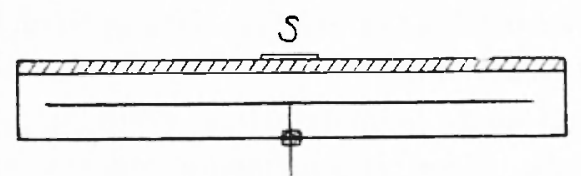

Fiv. 11 mente dispo-le -ulla rameras riatemal delle quali rentiene un sale di Radio in equilibrio eon il Radon ed il deporilo stlivo. Samno in

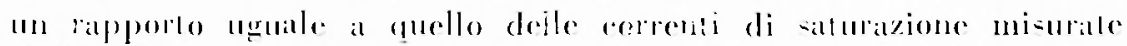
ron la prima e ri-petlivamente ron la ereomba ampollat.

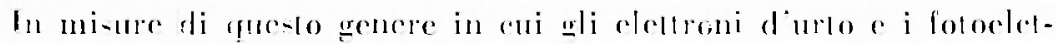

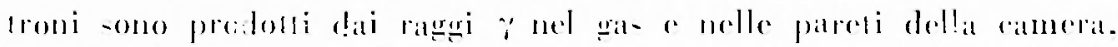

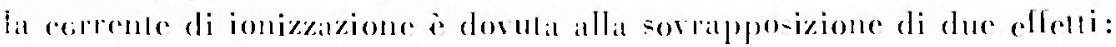
effelto "gaten ed effelto "parelen. E" evidente che guedo fenome. no di -ovalppo-izione is molo eomplese in quanlo non dipende

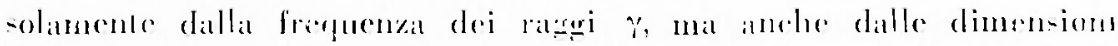
della camera e dalla nalura e dallo spe-ore delle pateli. 11 combi-

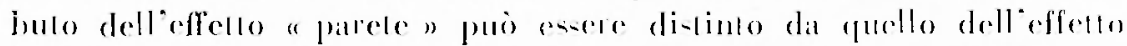

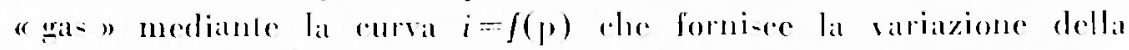
corente di ionizzizione in funzione della presione del was rontenutu

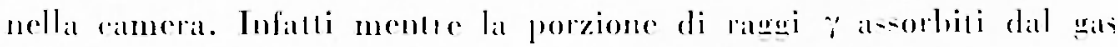
della camera is diellamente proporzionale alla presione del quar inizialmente la colrente di ionizzazione cesese meno raphidamenle di $f$ e diviene proporzionale alla presione solo dopo rhe que-tal hat ratgiunto un relerminalo valore.

Tale fenomeno dipende dal fatto rhe per quel valore della pres. sione lutli gli elettroni emesi slalle pareli sono completamenle an sorbiti dal gas sceso. In lali condizioni leffelto parete disiene ro-lanlie e un ulleriore aumento della presione produce solamente ma viriazione delleffello "gaten, il ruale dipende linearmente dalla pre-ione.

Lefletlo "parele "hat ha sha importanza nolle misture della ior- 
len-ilà dei raggi $\gamma$ e $A$ in puanto bata variate le condizioni -perimentali perche que-to effetto renda imponibile it ronfronto ira le mi-ure. Que-ta d la ragione per la quale la unila sli inten-ila dei lateri X. il "Roënlgen " -i definise rome la inlensila di un fazcio di ragri $X$ analizzati. ehe da orighine al nna corrente di ionizazionr, donula unicamente all"effello gas. di 1 u.e.s. p. $\mathrm{cm}^{3}$.

1. - Il conteggio delle singole particelle emesse da una sostanza radioalliva, la rui importanza fondamentale non ha bisogno di parlicolari illustrazioni, si otliene ron il contatore di Geiger-ytiller (14) nel quale la ionizzazione prodolla dal passaggio di una singola particella viene enormenente amplificata dalla ionizzazione secondaria. Ena uleriore amplificazione può ragriungersi ron un si=lema a una o due valvole termoioniche in modo rhe il pasagergo di una singola particellat può asere registrato da un romune numeratore mereatjuiro ( $(1 ;)$.

Velle sue linee essenziali il rontatore e coslituilo da un eilindro metallico. lungo il cui ase is teso num filo. generalmente di Tungsteno. con un dianetro di $0.1-(1), 5$ mm. Il cilindro metallico può eseres rhiuso ai due estremi da due lappi melallici ron supporli isolanti per il so-tegno del filo oppure ambedue ghi eletrodi posiono essere racehiusi in un involucto di vetro ( $v$ fign. 12). Il rontalore viene riempilo ron un gra-o una miscela di gar. la rui natura varia a seeondat

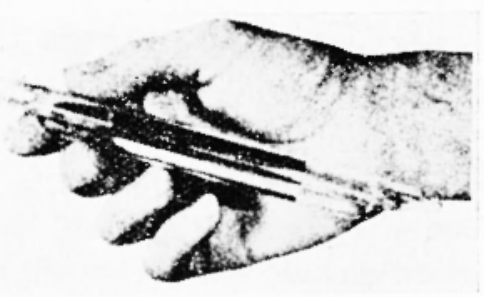

Fig. 12 degli -ropi rhe i propone leeperienza, adt una presione rompresa fral 2 e 111 rm Họ. Ad ecompio pes il contegrio di neutroni lenti -i usia come ga- di riempimento il $B F_{3}:$ iil lal rato la scatriva del contatore o dovula alla ionizzazione prodotia dalle particelle a eme-ene nellia mazione:

$$
{ }^{10} B+{ }_{0}^{1} n \rightarrow{ }^{i} L i+{ }^{1}
$$

Lat tensione applicata ai due eletrodi (1006-7.500r) a regolata in maniera rhe la reazione di almeno una coppia di ioni da parte della particella ionizzante, patsata altraverso il contatore, sia suffi. ciente ad iniziare la scaricat. E indispensabile che la scarica cosi 
immesalat i inlerrompal aulomaticamente dopo un tempo hrevisimo, in modo da consentire all"apparecehio di regi-trare la particellat =me. cessiva. Le cause di queslo fenomeno sono alquanto compleses: pe: la loro disersione e per la esposizione delle condizioni indi-pensilbili alla - la realizzasione rimandiano a un lavolo di uno di noi ( la rurva di fig. 13 rappresenta la rariazione del numero di im. pulsi regi-lrati, solto l"azione di una radiazione costante. al variare della lensione $V$ appliabla al contalore slesso. Dal es-at si deduec l'e-i-lenza di un intervallo abha-lanza ampio di valori della tensione per il ruale il nu. mero degrli impulai regi-lrati -i mantiene co-lante (regione di Geiger-Miiller). In rueste eondizioni $\therefore$ puo allermare rhe pratticameme tutle le parti-

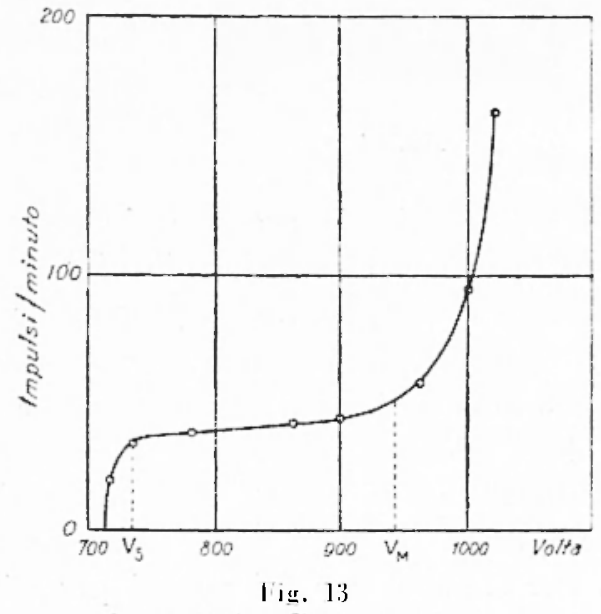

Caralteristica di me contalore relle ionizanti entrate nel rontalore rengono regi-trate. Regolando opportunamente la lensione applicatia al contalore si pui ollenere whe il numero totale di ioni prodoti all interno di questo sia proporzionate al numero di ioni primari. Un rontatore di questo tipo. delto combare proporsionale $\left({ }^{16}\right)$ o di Geiger klemperer per di-linguerlo dal rontatore di Geiger-Müller di rui abbiamo parlato finora, ejarlicolarmente adallo in tuite quelle riecole nelle quali si dehbano rontare solo partirelle v. in presenza di radiazioni $\beta$ o $\gamma$. La comsideresole diflerenza, aisente fra la ionizzazione prodolla dalle particelle e e fuella dei rawa

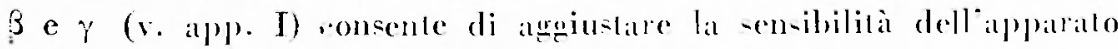
regi-tratore in maniela da contare solo le particelle a.

Tì rontegrevo delle particelle ionizanti a breve percorso guali quelle emesse dalle sostanze radioallive richiede ladozione di pareti molto ottili per ronsentile la penetrazione delle particelle all"interno del contatore. A lale scopo si rostruicono rontalori muniti di una finestra di mica (x. fig. 14) o di veiro soffialo (1i) il rui polere

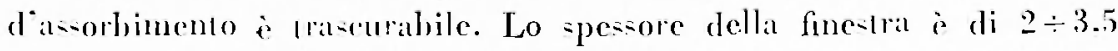
mor em². Altri ronlatori hamno le pareti di velro molto sollile rive- 


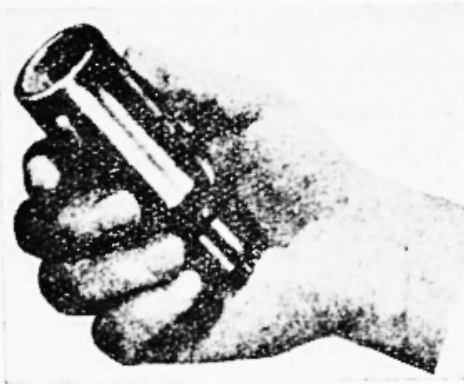

Fig. 14

stilo internamente da no stalo melallico, ollenulo per evaporizione: oppure di aguadag (sospensione colloidale di grafite in alcool). Recentlemente -ono sali costruili contalori ron un rive-limento di alquadar alle- Lerno $\left({ }^{1 n}\right)$. Le calralleristiche di funzionamento di tali contallori, rle -ono - late -ludiale da uno di noi ( (") - -ono tisultate pienamente soddisacenti.

5. - Stabilire la presenza, frequenza o variazione temporale o d'altro tipo degli clementi rationtrivi nella terat nelle actue e nell'atmo-fera é compite the si propone la Geofisica.

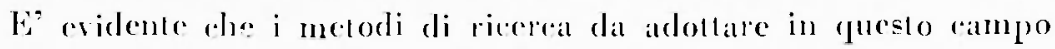
debbono oricntarsi verso mi-ure rapide e basale sull'analisi di c trac-

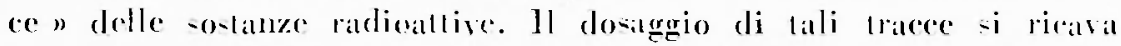
greneralmente dalla mi-nat della fuantita di Emanazione clue -i e formala come prodollo della disintegrazione della sostanzal da dosare. Per esempio uno dei metodi per misurare la quantita di Ratio

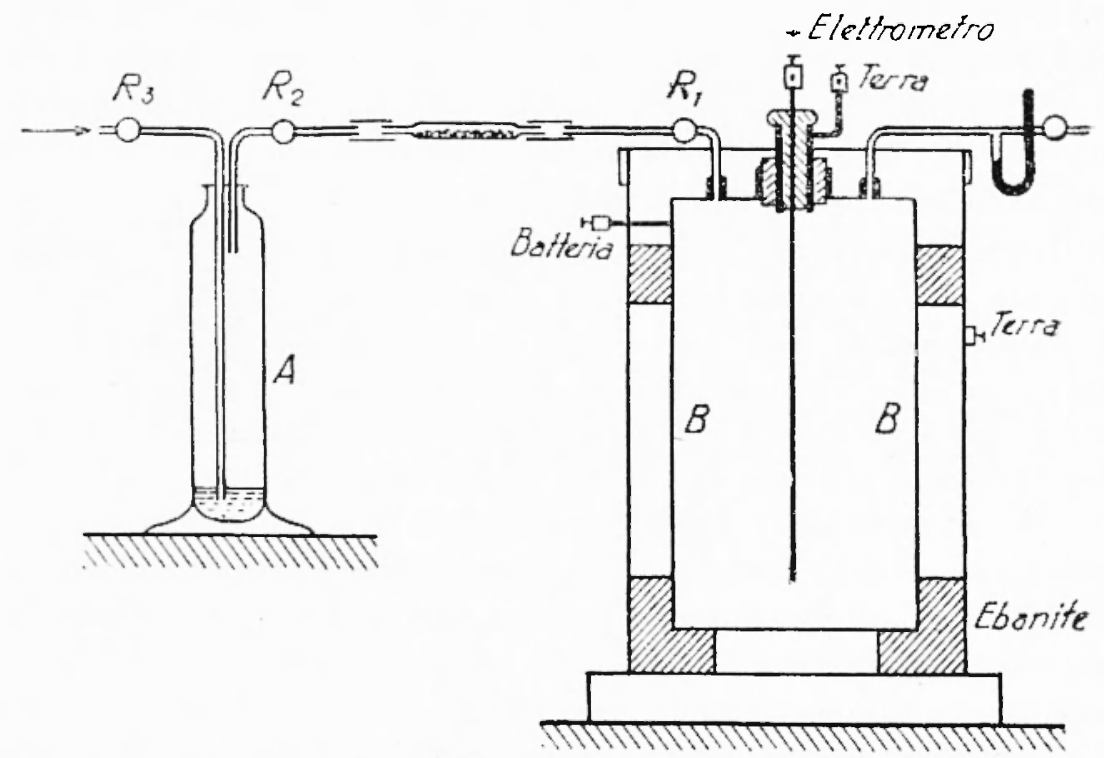

Fig. 15

Dispo-itivo per lialrazione del Radon a freddo 
conlenula nei minesali și Tranio consi-te nel polverizzale mal pir-

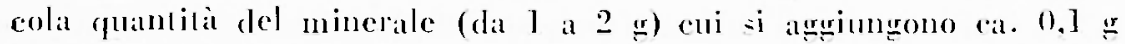
di clormo di Bario. Suecesivamente con un opportuno trillamento rhimiro si estrae il Bario radileto del quale si prepara una soluxione, che -i introdure in una boceia, $A$, a due vie $\left(R_{2} . R_{3}\right)$ rollegalia allataverso $R$, eon mulanera di ionizzazione $B$ di dimen-ioni grandi in

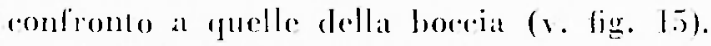

Prima di iniziare la misura si aspira il Radon rontenuto nella soluzione con una pompat poi si rhiuclono i rubinelli e si la-cia atecumulare il Raton per un periodo di lempo. variabile da une a lre griorni, maseor $=0$ il fuale si mntroduce il Radon nella camera di ionizzazione ron una seric di aspirazioni suecesive. Come lempo di acrumulazione si assume la diflerenza fra listante di rhiu-ura del rubineldo e loistane in eni c leminala l'aspilarione. Queste misure

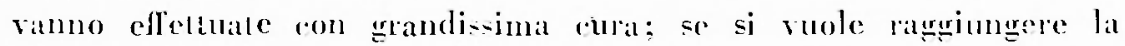
massima precisione con-entita dallesperienza (15.5\%). Per il dosagtrio delle sostanze radioatlive contenute nelle alcopue minerali naturali si usa un melodo legrgermente diverso. Jáachual da maminare viene introdolla in un pallone da 1 a 31 ex $s$ portata allat chollizione in maniera da scacciare il Radon, in essa conlenulo. Sucre-iramente si chiude il pallone e si allende che il Rialon -i areumuli per un

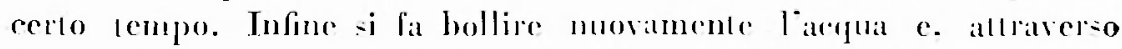

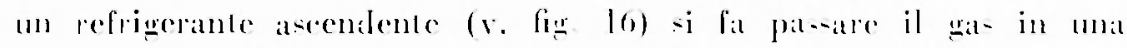
valncia di ionizzazinne.

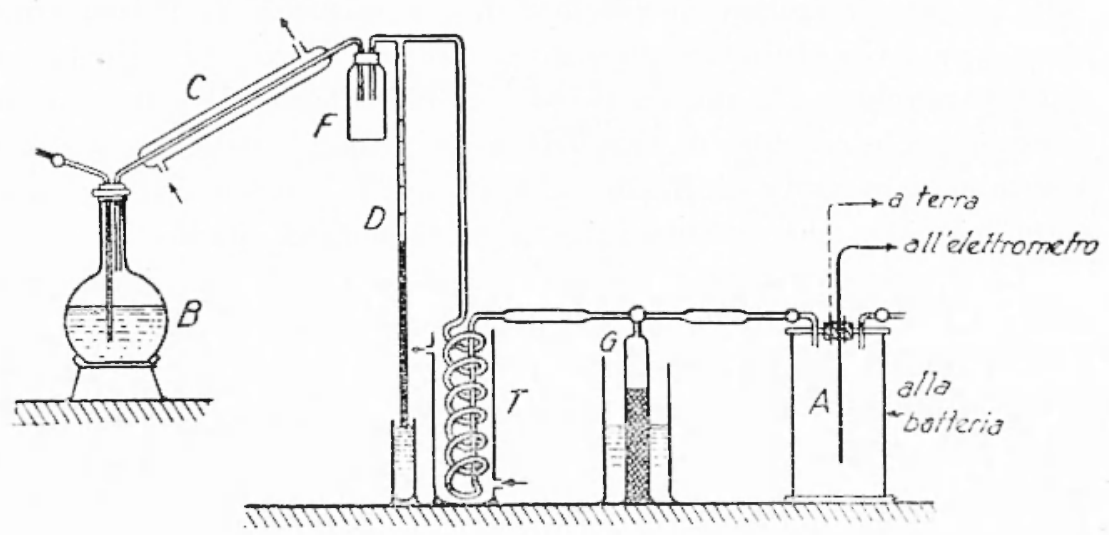

Fig. 16

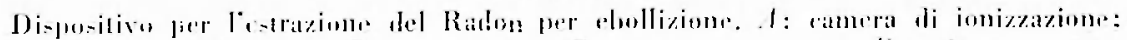

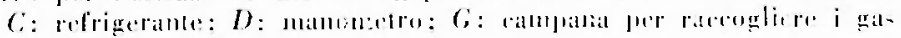


I metodi soprade-rerilti -ono adalli all"esame del Radon (v. alpjendie IV). Lo sudio del Toron e dell'Aelinon is reos asali più difficile dalla berila della vila di queste due sostanze. In yueslo caso anziché ricomere alle soluzioni si preferiece partire da sostanze solide (per es. idralo di Torio). Il Toron viene aspirato in ma camera di ionizzazone insiems con l'aria rhe rireonda la materia produllrice

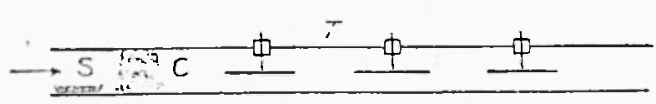

Fig. 17

$\therefore$ :orqunle: $C_{\text {: }}$ tampone di colone oppure lo si traseina lungo un mbo $\mathrm{T}$ contenenle elelrooli equidistanti (r.fig. 17) 1. -i misura la rorente di altuazione cle passa lra

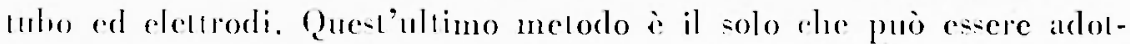
lalo nel ca-o del dosagrio dell' telinon rhe ha una vila media ancora jiin breve (').

11 dosagrio della Emanazione si rieara dalla misma della corrente di salurazione rle esa produce nella ranera di ionizzazione in eui $i$ slata introdolla. Questa corrente dipende dalle dimensioni * dalla forma della ranera ossia dalla mincre o mageniore ulilizat zione delle particele e emese dalla Fmanaizione.

Inolue la corente o fumzione del lempo quindi is indispensabile preci-ale listanle nel quale se ne offellua la mi-mra. In cenere si

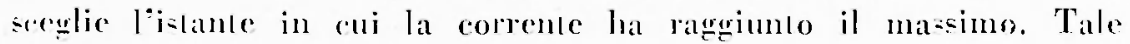
istanle corrisponde alla completa formazione del deposito atlivo. Poihis il massimo i piullose piallo, la misura può essere iniziala lre " quatlro ore dopo l'introduzione del gas nella camera.

Con que-to melodo :i poseno dosate ruanlitì di Radon romprese flat l lu mnificalo arsoluto alla mi=ura è indispensabile larare la camera os-ia delerminare la rortente di saluratzone che produce nella ramera una eleleminata fuantiaj di Radon, per es. fuella emesea da una soluzione rampione che ronliene una quantili nola di Radio.

Lal deleminazione del contenulo di Radon nella aria si consegue anche dall'esame delle radizioni emesse dal deposilo allivo (v. ap)iendiec Th) il principio su eni si hasia lale misura i il seguente: - 11 lun condultore carico negalivamente che si lrova in un anbiente contenente Radon si deposilano $\vdots$ nuclei di Radio A. Introducendo il rondullore in mat ramela sli ionizzazione si può decominare il secadimento del Radio $A$ ivi depositato. Da $e s=0$ si può risalire alla -oncentrazione dellatlivalome (Radon) purehe si abbia la rerleza -he: 1) il depo-ilo sia co-liluilo micamente da Radio A: 2) lulto il 
Radio A formatosi in conseguenza, della disintegrazione del Radon da dosire si sia depositate sul condultore. I metodi fondali su questo principio sono stati adottati nelle ricerehe di Geofisica printa ancora dei metodi direlli che abbiamo esposti pocinzi. Quanlungue essi siano parlicolarmente semplici la loro incerlezza è peró tanto con-iderevole che, ovumpue sia posibile, si preferisec sosituirli con i metodi dietli. Buoni riwultali sono stati conseruili col metodo di G. Aliverti $\left(^{-0}\right)$ nel puale sono stali introdolti alcuni particolari ate corgimenti, in modo da rendere lo slmumento mağgionnente sensibile.

La rivelazione e la mi-ura di deboli radiazioni è ostacolata c condizionata dalla ionizzazione residua dell’apparecehio. Tale fenomeno, eni abhiamo wia anecnnalo in precedenza ra allribuilo a cause diveres. Prima di lulto allazione dei ragegi $r$ contemuti nelle paredi

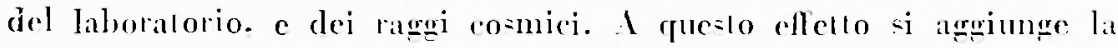

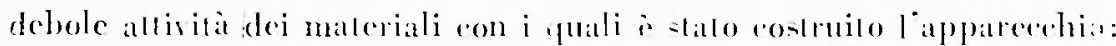

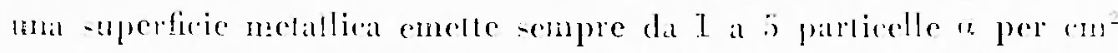
per rara. Evidentemente leffetto della ionizzazione residua rostitui-ne una specie di "fondo " che compare in lu!le le misure di ionizzazions. L'elletto di fondo può essere ridbotto enn=iderevolmente con un dispositice a compenasione $\left(^{-1}\right)$, costituite da due camere di ionizza-

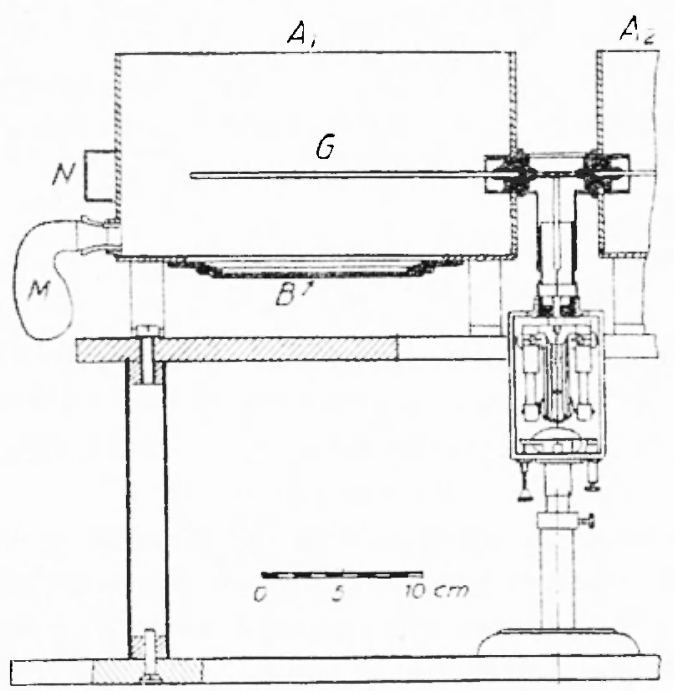

Fig̣. 18

A: frima ramera di innizzazione: 1 : seconda ramera: $B$ : -litla: $G:$ eleltrudo centrale: $M:$ tubo pre l'e-cicante: 1 : caviti per il campione xione perfellamente $11-$ mnali i rui elellrodi renlrali sono rollewati alla semo eleltrometro. le remsioni applicate alle patreti delle due camere sono lyanuli o di sengno opposto. dimodoche l'elettrometro mistria la differenza fra le due corene di ionizzazione. Ial so-lanza da e-aminare puo e-sere introdotla nelími o nellaltra delle due ramere mediante un -islema di slitte interamloi:tbili (s. fir. 18). La ror. rente di ionizzazione do- 


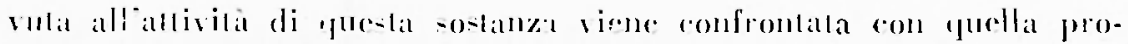
dollat dai raegri $i$ emesi ha un eampione -i-lemalo in ma delle dus

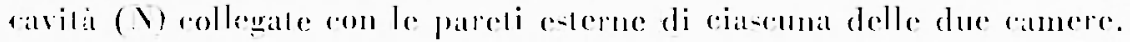

Indichiamo ron $A, B$ ed $S$ la corrente di ionizzazione dovula rispeflivamente alla radiazione in comes alla ionizazione residua e

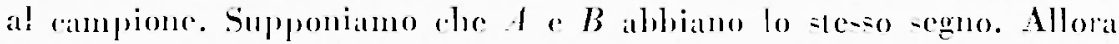
se il rampione $a$ siluato vieino allal rameral nellal quale si troval la

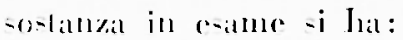

$$
S \div A-B=C / 1_{1}
$$

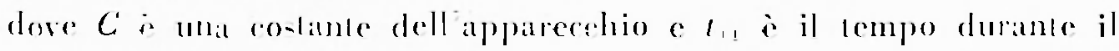

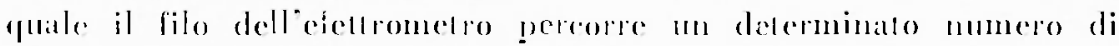
divi-ioni. Tratpontando il rampione atranto all"allrat ramera si otliene:

$$
S-1-B=C / l_{1}=
$$

dove $t_{12}$ i il tempo corrispondente.

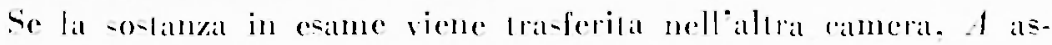
sume segno oppo-lo a quello di B. Ripelendo il trasporto del ampione effellualo nella misura precedente s otlengeno altre due equat zionil:

$$
\begin{aligned}
& S-1+B=C: l_{21} \\
& S \div 1-B=C \cdot t_{2}
\end{aligned}
$$

Dalle qualtro equazioni $[1],\left[1^{2}\right]$. [2] $\left[22^{\prime}\right]$-i deduec una e-pres. sione de!la allivila della so-latnza in centue dallat fuale es salo climi. nallo liefrello della ionizalzione serdua:

$$
A=\frac{S}{2}\left\{\frac{t_{12}-t_{11}}{t_{12}+t_{11}}+\frac{t_{21}-t_{22}}{t_{21}-t_{22}}\right\}
$$

Con que-lo melodo epo-ibile mi-mrate la allivila di una quantila

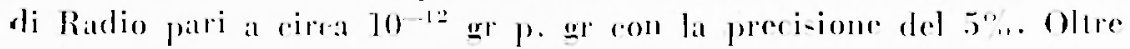

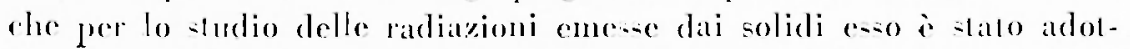

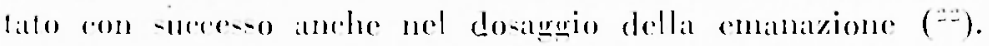

Areatlo alle misure effelluale in laboratorio su campioni di roce

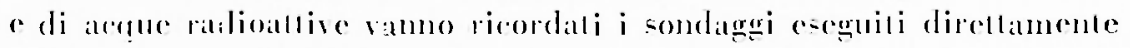
nei porsi pelrolifreri. Questa eplorazione not: sollo it nome di " ca-

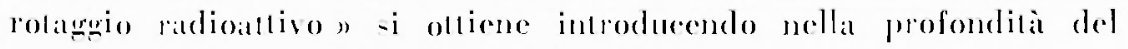
pozzo mono strumento rivelalore di partiectle ionizzanti, sestituilo da

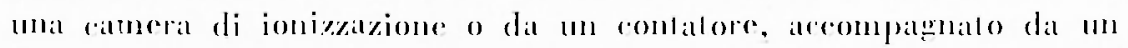
comseniente -iscma de registrazione. Per mat eposizione particola- 
reseriala di questo metodo rimandiamo alla rasegna pubblicata ultimamente dai dothori Quereia e Tribulato ("-1).

6. - Tulle le mulà di misura adoperale in Radioaltivita si riferi-cono al Radio rhe ron relativa facilila può ottener-i allo stato puro in quantita stfleiente. Il campione di Ratdio i co-lituito da 21.99 mgr di Radio purissimo di-idralato che dallatgo-to 1917 si trova nel Bureau des Poids et Mé-ures di Parigi. Nelle ricerder di Geofesea hat grande inlerese considerare lat quantia di Radon in rapporlo

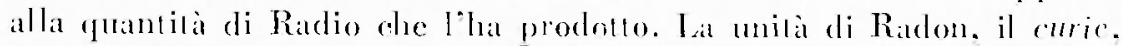
à definila come la quantila di Radon in equilibrio con l y di Radio. Mole, w-illi sono i multipli a $i$ soltomultipli di que-ta muta (me. ! $(\mathrm{C} . .$.$) .$

Gli ordini di grandezza rhe sincontrano nelle ricerehe di Geo-

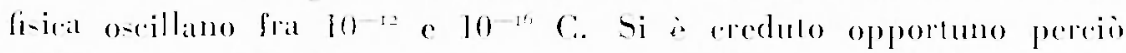
defunire una mila di concentrazione lo "Emann n, os-ia la concen-

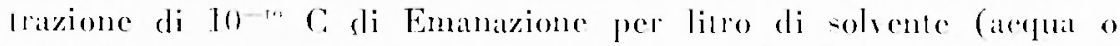
aria). Le milà wate più frepuentemente sono rateole nellat Tabella III c nella Tabella IV $\left(^{-1}\right)$.

TABELAA III

\begin{tabular}{|c|c|c|c|c|c|}
\hline & C & $m c:$ & $11 \mathrm{C}$ & $m ! 1 \mathrm{C}$ & U.E... \\
\hline Curir & 1 & $10:$ & $11^{1 ;}$ & $11^{\mathrm{9}}$ & $2.767 .10^{6}$ \\
\hline Nillieurie (me) & 110 & 1 & $111^{*}$ & $11^{6}$ & 2.867 .10 \\
\hline Microcurie 1116 & $10^{-i}$ & $10:$ & 1 & $10^{\circ}$ & 2.76 \\
\hline Millimicrocuric (m!) (c) & $100^{9}$ & $71^{-6}$ & 10 & 1 & 2.865 .110$. \\
\hline Corrente rquival. ILE..s. & $3.615 .10^{-4}$ & $3.615 .10^{-1}$ & 0.3615 & 361.5 & 1 \\
\hline
\end{tabular}

Tinfila IV

\begin{tabular}{|c|c|c|c|c|c|c|}
\hline & (./] & แ11 $\mathrm{C} / 1$ & $\mathrm{C} / \mathrm{]}$ & mụı: 1 & Emian & Mincher \\
\hline$C / 1$ & 1 & {$[11:$} & $10^{15}$ & $10 !$ & $10^{111}$ & $2.767 .10^{10}$ \\
\hline$m c / 1$ & 10 & l & $10^{\circ}$ & $11^{15}$ & $10^{7}$ & $2-56.710^{6}$ \\
\hline $11 \mathrm{C} / 1$ & 10 & 10 & 1 & 10 & $10^{\prime}$ & $2.76 \pi .10$ \\
\hline$m ! n(: / 1$ & $10^{-9}$ & $10^{\circ}$ & 111 & 1 & 10 & $2.76 i$ \\
\hline Eman & $70^{111}$ & $101^{\circ}$ & $10^{-1}$ & (10) & 1 & $11.2-26$ \\
\hline Marke & $3.615 .10^{-21}$ & $3.615 .10^{-}$ & $3.615 .10^{-1}$ & 10.3615 & 3.615 & 1 \\
\hline U.K.S. 1 & $3.615 .11^{-7}$ & $3.615 .10^{-4}$ & 0.361 .5 & 1361.5 & 3615 & 10 \\
\hline
\end{tabular}




\section{APPENDICE I}

Il fenomeno della radioattivili $<$ dovulo. rom noto. al fillo rler i muclei deghi elementi di numero alomico -mperiore a 82 (b) sone insabili ossia in grade di tra-formatrs spontaneamente in un alloo nucleo. Tale tra-formazione pàò arrenire secondo due processi. In uno di fue-li - emi-sone di parlicelle a - si rerifua la espulsone di frimmenti dell'zedifreio nucleare. Come dimostrò Rullerford nel 1903 le particelle a sono nurelei di Filio. il secondo elemento del sisema periodico $(Z=2)$. Cosicche con la perdita di ma particella " lis rarica nurleare i rishue di $+2 c$ e, ronseguentemente. l'elemento $\therefore$ trasformat in queilo rle lo precede di due posti nel sistema pe. riodico. ?er es. il Polonio $(Z=81)$-i rastorma in Piombo $(Z=82)$.

l'allro proceson - disimegrazione - consisle nella emissione di celenoni reloci. Conseguenza di que-lo processo è un aumento pari a te della rarima nucleate (in corri-pondenzal alla perdita di mal rarica -r) quindi la tra-formaxione dol mucleo in quello de lo serae nel sisema periodien.

Duranle l"uno o l'aluro di quesli processi può rerificarsi l'emi:-

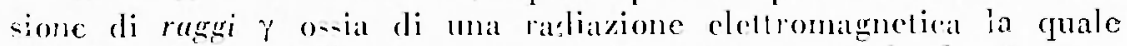

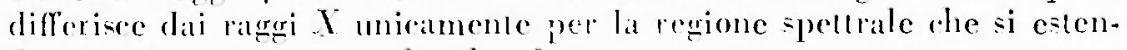
de malgriormente rerso le alte fierefenze.

I. "anali-i delle tre ratiazioni a, $\beta$ e $\gamma$ si effollua mediante l'esame dellas=sorbimento delle radiaxioni tese nella materia a della deviazione dre esece subiseono sollo lazione di m ampo magnelieo. Lal renergia lolale della radiazione a nella mal-ima parte dei rasi is ren-iderevolmente superiore a puella slei rageri s e $\%$. Quando in ma ranera di ionizzarone di dimensoni norinali (*os) si esiminal l'azione ionizzanle delle lac radiazioni $u, j$ e $\gamma$ emeso da ma o più sonlanze

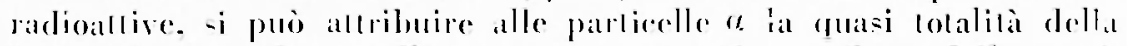
ionizzazione prodolla nella camera, mentre il contribulo delle parti-

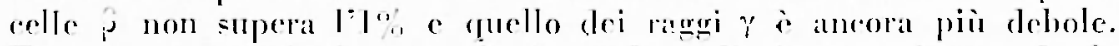
'Tutavia -i può fareilmente eliminue la ratiazione a rieoprendo lat sorgente con uno sehermo sollile (p. es. basta un forlio di $\Lambda l$ dello

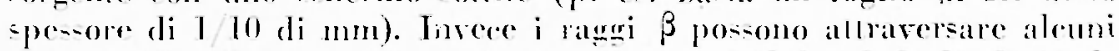
mm di $A l$ e l'azione dei latreri $\gamma$ è ancora sen-ibile al di la di qualche rm di Pb.

\section{APPF SDICF, II}

Lordine di grandeza dellal velorila iniziale ron eni le parti-

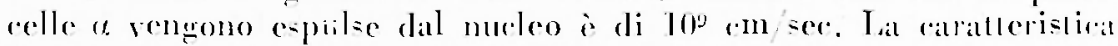
fondamentale di questa radiazione è restituila dalla maniera hru-cat

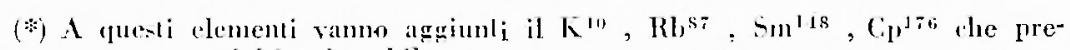
sentano pure una allività misurabile. pareti.

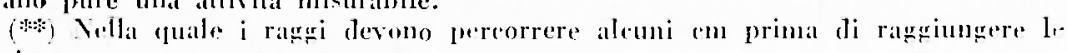


con cui esa viene assorbila dallearia del mat certa di-lanza dalla sorgente. Tale distanza ha m valore ben defnito dipendente dalla nalfural dellat so-lanza radioalliva da!la fuale vengono emesso le particolle. Inoltre la trateltoria di ciatem raggio i rellilinea e. se la solsrente è costituita da uno strato latlio soltile da critare l'assorbimento

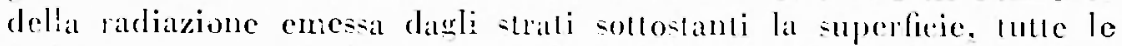
trabllotic sono pressa poco erruali (r. fir. 4). Que-lo fenomeno sta ad indicare che la :elocila con rui le partieclle a vengono enesse dat

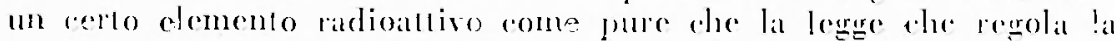
perdila della loro enereria durant: il pa-singio allraverso la malcrial - la -les-al per tulle le parlicelle. Dallo tudio sisemalico delle parlicelie remisponde un solo gruppo di particelle (h. Aleuni radioelementi emeltono però una radiazione e compleses compo-la di parecehi gruppi rhe differianono per la veloetil di emissione e pere il apereorso".

lià numerosi e complicati sono i fenomeni che -i verificano durante il palsagrgio degrli eleltroni atlraverso la maleria.

A causa della piceolezza della sua massa leelellone e in grado di traferire sran parte della proprial enereria e del proprio impulso

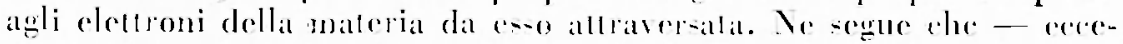
zion fatla per il caso di chergie assai elevate - geli elellroni compions percors piò o meno jregolati con frectuenti cambiancnti di dirczione dovmli agli unt subiti da parte degli eletroni e doi nuelei

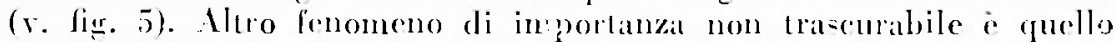

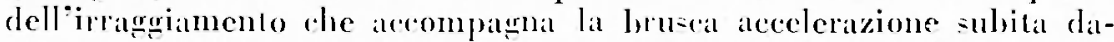

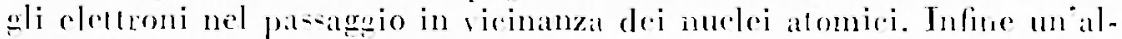
tra diffeolà non rascurabile nollo suclio dei rarge $\beta$ è co-tituita dal fallo rhe la energia delle patticelle $\hat{p}$ emese dalle so-lanze radioallive

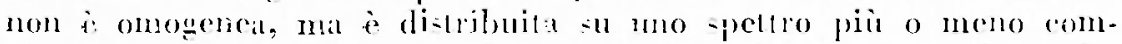

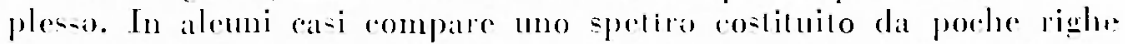
di esergia ben defirita (lid fe), in alui mo spettro continuo (RuE). in allei amora uno spelto risulante dalla sovrapposizione dei due ( $R(B)$.

1 ragrgi $\gamma$ gencrano lungِo il loro pereorso radiazioni -econdarie, di natura electromatrneliea e rolpuseolare: i fenomeni ai quali ra attribuita la formatzione di lali raggri sono esenzialmente l'effetto folocintrico e la diffusione Compton. La eomponente eleltromagneticis della radiazione sccondaria è costituita dai ratresi $\gamma$ diffusi e dalla radiazione di fluorescenza, mentre la componente corpuscolare è formala da foloclettroni, eletroni d'urto e coppie di clettroni positisi - negativi. Ta conereria individuale degli eleltroni serondari aresce con la frequenza. Per es. quando ragere di frequenza mollo eleratal $(\lambda<200$ (X) atlrarersano corpi leggeri l'assorbimento fotoelellrico e trascurabile nei eonfonti della difusione, cosieche lutti i ragre $\gamma$ secondari pos-

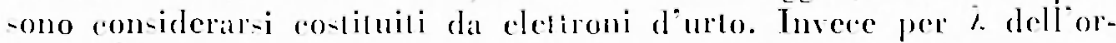

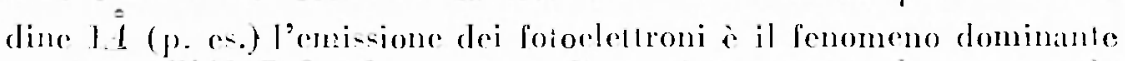
porino nell" Al. Infune la creazione de roppio compare solo per eneroie 


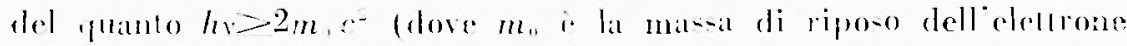
- $c$ la velorila della lureo.

\section{APPENOICF III}

Se ron mo opportano dispo-itivo perimentale $-\mathrm{i}$-rewe la ero-

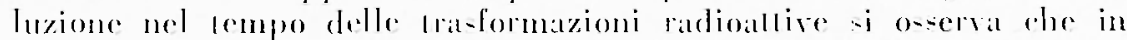
un delominalo intervallo di lempor degli $Y$ alomi ronlentuli in $m$ w d) mal -o-tanza radioalliva, i disinlegrat un numero indipendente dalle partieolari rondizioni fi-irhe e rhimbehe dellat so-tanza e della

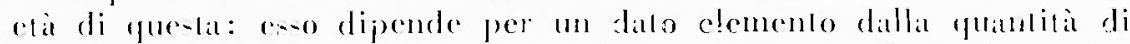
-o-tanza presente e precisamenle o proporzionale ad V. La legege dhe

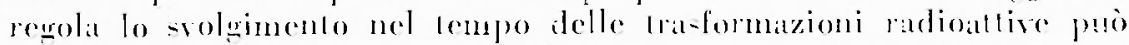

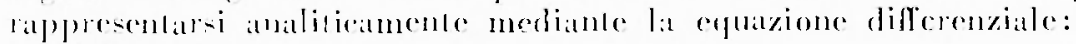

$$
d V-i d e
$$

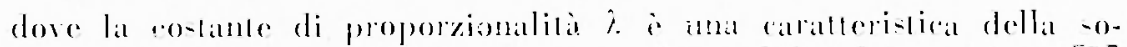
slanza. deta costante di disintegrazione. Per -labilire ha equatzone [1]

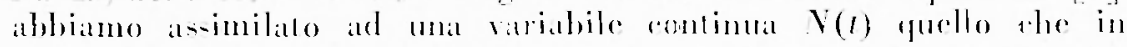
realla is un numero intero, dimodorh: fulte le considerazioni nelle yuali inlerviene lat [1] valgomo solo se il numero degli alomi asere vali o ellemamente grande. Lal $\mid 1 !$ integlatil divienc:

$$
\left.M(1)^{--}\right)+i
$$

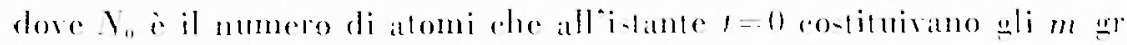

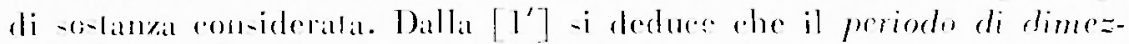

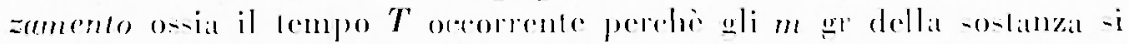

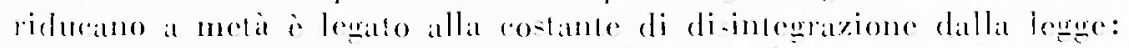

$$
\hat{t} T=\lg _{-2}{ }^{2}
$$

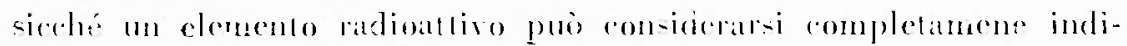

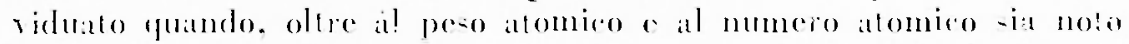
anche il periodo di dimezannento (o la rostanle di di-interarione).

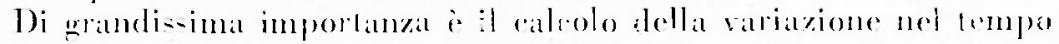
di un elemento radioallivo puando, durante lo srolgimenlo del sum proeceo di disintegrazione, eso viene rifornito dallat o-lanzat madre

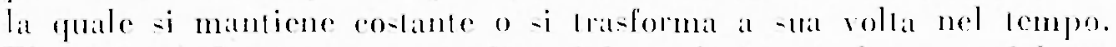

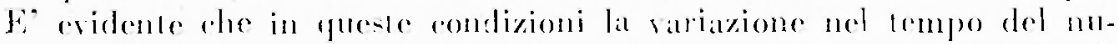

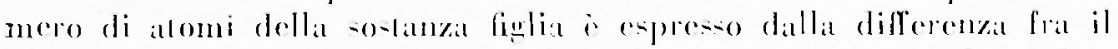
mumero di atomi che si dininterrano o il numero di atomi che -i fore 
mamo in consegunenzat dellat di-intearazione della sostanza madere di-

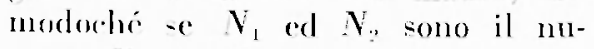
mero di alomi contenuli in mal de-

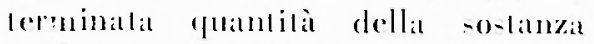
madle e ri-pellivimente della -o- Lamza figlia e ha e h... le corri-pondenli co-lanli di di-intewrazione, pos-iamo rieatratre dallar [1] il sislemal di equazione:

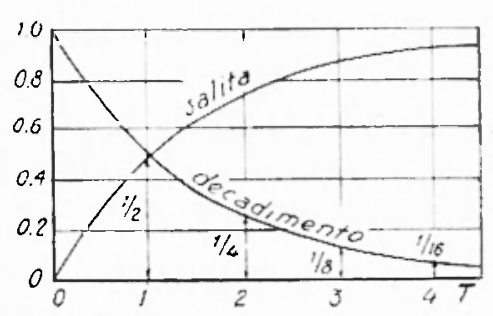

Fị. 19

$$
\left\{\begin{array}{l}
d V / d=-i_{1} N_{1} \\
d V / d t=-i_{1} V_{1}-i_{2} V_{2}
\end{array}\right.
$$

la cui soluzione generale i cepresa dille erfuzioni:

$$
\left\{\begin{array}{l}
N_{1}=N_{1}^{0} e-\lambda_{1} t \\
N_{2}=\frac{\lambda_{1}}{\lambda_{2}-\lambda_{1}} N_{1}^{0} e^{-\lambda_{1} t}+\left\{N_{2}^{0}-\frac{\lambda_{2}}{\lambda_{2}-\lambda_{1}} \mid e^{-\lambda_{22} t}\right.
\end{array}\right.
$$

E-aminiano due car-i partieolari ehe si presentano frepuentemente nei probleni inleres-anli fue-lal no-l rat ras-egnal:

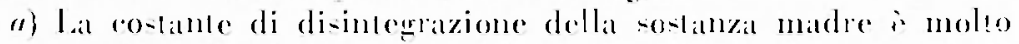

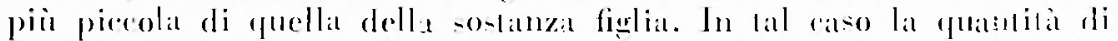
so-tatizat madre pre-ente durante la tra-formazione della so-lamza fịtial

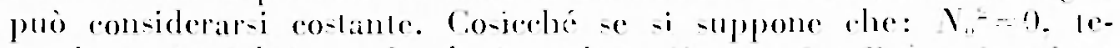

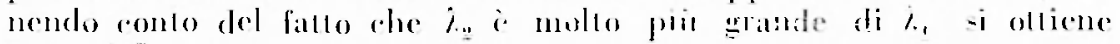
dalia [2] la relazione approwimala:

$$
N_{0}=\frac{\lambda_{1}}{\lambda_{2}} N_{1}^{0}\left\{1-e^{-\lambda_{22} l}\right\}
$$

Dop: un lempo molo grande anche la quantila di sostinzal figlia acensibilmente costante ed is uguale a: $i_{1} N_{1}^{0 / h_{2}}$. Fial le due -0-tanze i

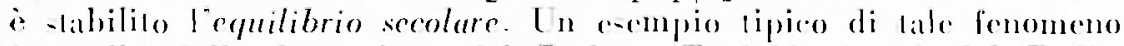
a quello della formazione del Radon $(T=3.84$ griorni) dal Radio

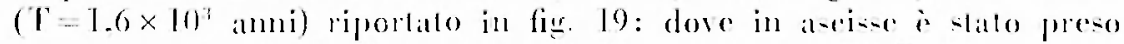
rome cempo milario il periodo di dinezanmento del Radio.

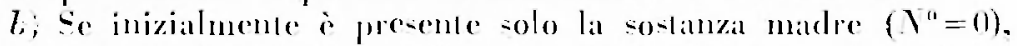

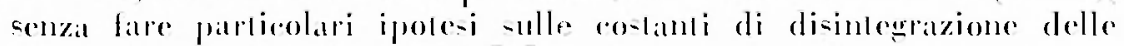
due -0-limze, si olliene dalle $\mid 2\rceil$ :

$$
N_{0}=\frac{\lambda_{1}}{\lambda_{2}-\lambda_{1}} N_{1}^{0}\left\{e^{-\lambda_{1} t}-e^{-\lambda_{2} t}\right\}
$$

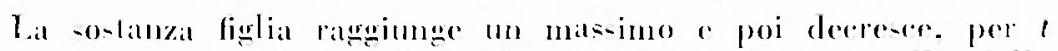
moles erande, ron il periodo della -ostamza mader o quelles della

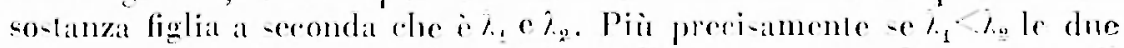
so-tamze deceseone con il periodo della -o-lanza madre mentre il loro rapporlo: 


$$
\frac{N_{2}}{N_{1}}=\frac{\lambda_{1}}{\lambda_{2}-\lambda_{1}}
$$

si mantiene costante. Si dice allora dhe fra di esse si è stabilito l'cquilibrio transitorio. Se invere $i_{1}>i_{0}$ per $t$ grande, la sostanza madre a sparita e rimane la sola sostanza figlia che decesce con il periodo proplio.

Un esempio dei diversi lipi di trasormazione si ha nella fig. 20 in eui è riportato il decadimento del deposito atbivo del Ratio nell'ipoleai rhe inizialmente fose presente il solo Ralio i puro.

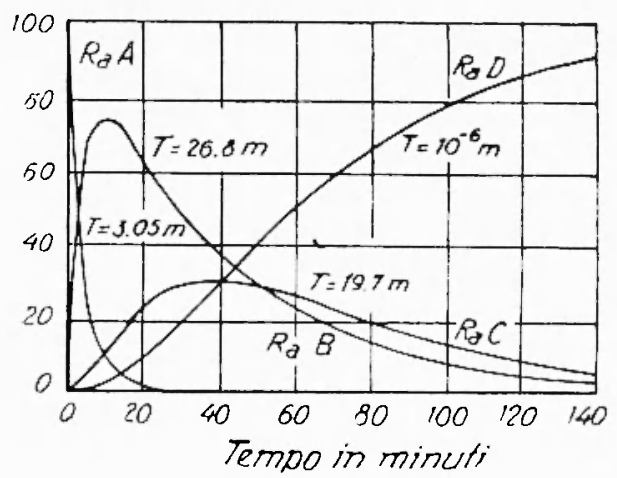

Fig. 20

Le regrole di spostamento rhe abbiamo enumcialo nella appendice I comentiono lat delerminazione de! pero e del numero alomico di tulli gli alementi di mu: scrio di trasformazioni purthé questi siano slali slahiliti per un clemento.p.es., il primo dellat serie. Enal serie di elementi rhe si trassormano sueresivamente l'mo nellablaro fino atd in nueles stabile si dice famiglia radroatriva, Le famiglie radiasallive ronoudute sono tre: dell't ranio-Radio. del Torio a dell' nio, ed hamno rispelisamente come aposipile: l'Tranio $(Z=9 \cdot 2$,

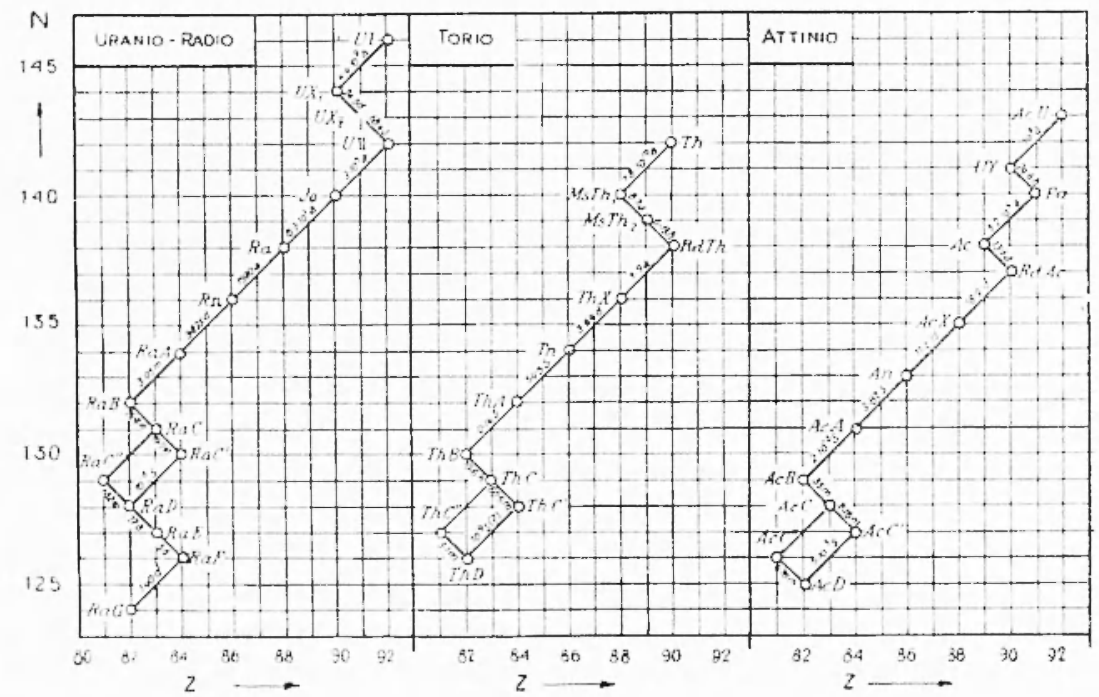

Fir. 2 I

Fandiglie ratlioaltive 
1=238, $T=4,5 \times 10^{7}$ anni), il Torio $\left(Z=90, A=232, T=-4 \times 10^{10}\right.$ anni) e un isolopo dell'Uranio ( $\left.{ }^{25}\right)\left(Z=92, A=235, T=7.13 \times 10^{\circ}\right.$ anni) molto raro in nalura (abhondanza pereentuale 0.720 ).

II prodollo finale non radiontivo is per ciaseuna delle tre famiglie un i=olopo del Piombo.

La disposizione dei singoli elementi che compongono le tre filmiglie radioatlive insicme con le trasformazioni che portano dall uno all altro elemento di ciasoma famiglia somo illustrale nella fig. $21\left(^{(-1)}\right.$ dove in areisea indiealo il numero alomico $Z$ e in ordinala lat diffe-

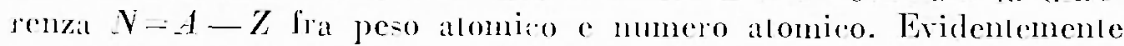
ogni disintegrazione e posta lelemento di due posti rerso sinistra e due posi verso il bako. orni disinlegratione $\beta$ di un po-lo verso destral ed moro reroo il basso.

\section{APPE NDICE IV}

Fra gli elementi radioatlivi rompare un solo elemento allo slato gasico-o. Fimanazione, di numero atomico 86. Fsso ha le proprieta thimiche di un gra raro e può esiere consideralo come un omologo superiore dello Xenon. Lat separazione di fueslo gas dalla sostanzil

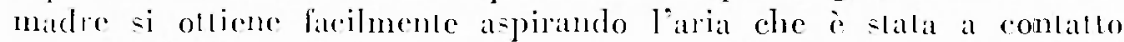
ron fues ultima. Cosi i posono allenere allo slato puro i prodolli sucresiyi i quali costituiscono il deposito attivo composto dai eolpi A. B, C. $C^{\prime}$. $C^{\prime \prime}$. solidi. di periodo piutlosto here. Liorigine di queso nome ra riceralo nel lalle che gli alomi formalisi in ronseguenza della tra-formazione degrli atomi di Emanazione limangono so-peri nellatria come una polvere estemamente sollile the si fisia

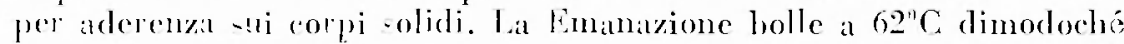
s puo fare uso dellaria liquida per conden-arla. Si seioglie nei liruidi in differente misura. Il rapporlo fra la concentrazione della Emanazione (in equilibrio) nell atequa e rispetlivamente nell'aria 0 sia lar epresione:

$$
u=\frac{\left.v_{\mathrm{a}} E m_{11_{2}}\right)}{v_{1 \mathrm{t}_{2}}, E m_{: 1}}
$$

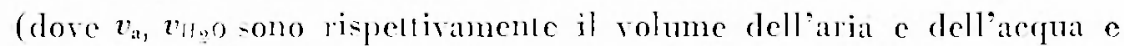

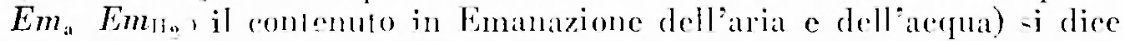
-olubilila e dipende semsibilmente dalla comperalura. Vella labella 5 riportiamo lat -olubilita della Emanazione in rarie ostanze.

\section{TABELLA V $(\because)$}

Olio di oliva e afhini $\quad . \quad$. $\quad . \quad$. $\quad 28$

Solfuro di Cillyonio $\quad . \quad$. $\quad . \quad 23$

Trementiua, Elere Elilico 


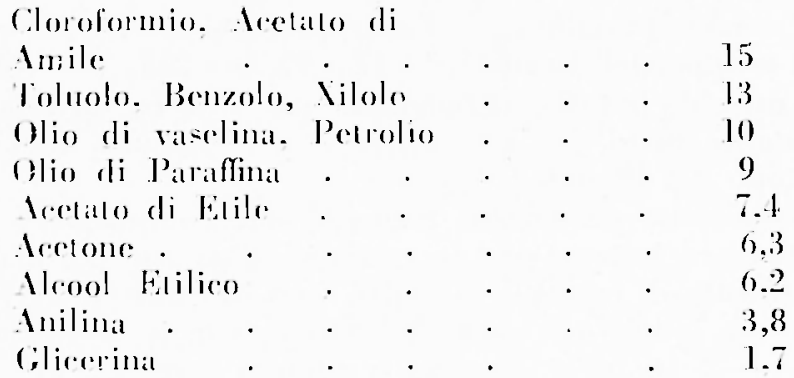

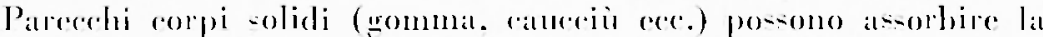
Fimatuazione, mat que-lo fenomeno is partieolatmente esaltalo nel catrlone.

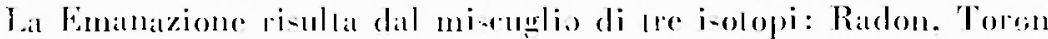
al Lelinon, tulli e tre radioaltivi u. Il primo deriva dal Radio e - i

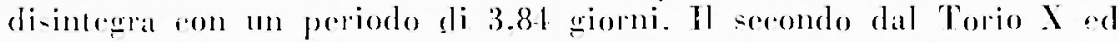
lat un periodo di 5.5 secondi. Il periodo del remo ro proviene

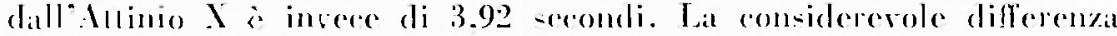
fra 1 periodi di dimezzamento di que-le lre so-lanze semplifira noferoluente il proce-so di separazione della prima dalle seconde.

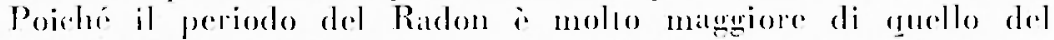
- mo deposito allivo, dope aleume one il Radon is in erpuilibrio ron

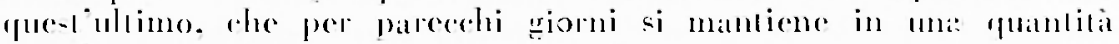

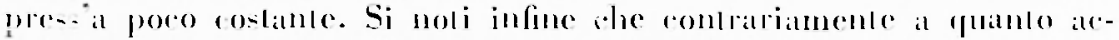
cade per le altae due Emantazioni il depo-ilo allivo del Rabon non -i lra-forma immedialamente nol Piombur inallivo ma passa ancora

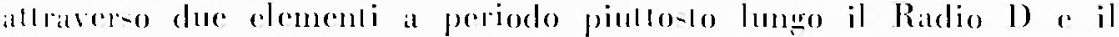
Radio: F (Polonio).

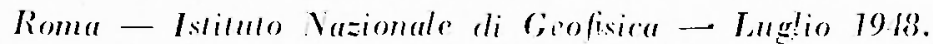

\section{RIASSUNTO}

Il presene lavoro costimisce la prima parle di una rassegna sai vari problemi interessatai la radioathivila della terra, che is stabu effemuata in relazione alla progenlazione di un laboratorio per ricerche sulla radioalliciti della terra presso l'Istitado Nasionale di Geofasica. la prima parle è dedicala eschusiramente ai melodi o agli strumenli da atoltare in simili ricerche. Es stata inolere corredala di una

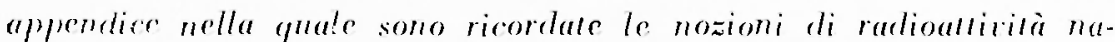
lawale che interenengono nel corso della tratlazione. 


\section{BIBLIOGRIIAI}

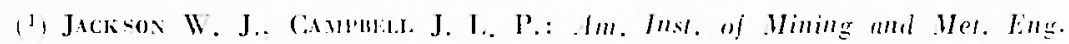
- 19.15.

1-1 Vervask W.: Les Problemes de la Radiogénlogie - Paris 1935.

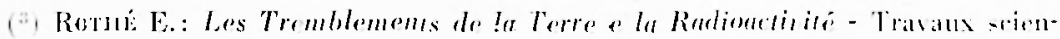
Lifigue. Pari- 1916.

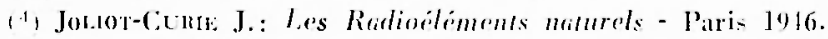

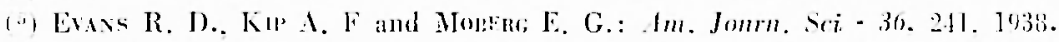

(") L km W. D. and Plober C. S.: Am. Journ. Sci - 93. 210. 19|?.

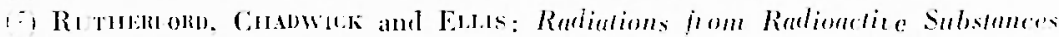

- Cambridge 1935.

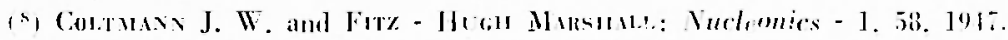

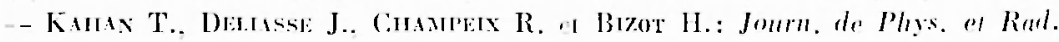
$-9,25,1918$.

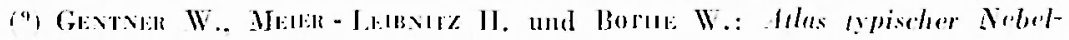
Liammarbildme mil... - Berlin 19.12.

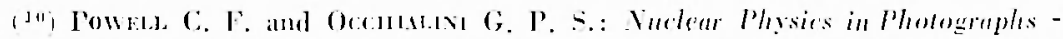
Oxford 1917.

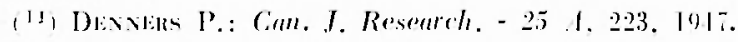

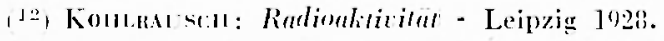

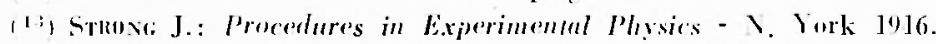

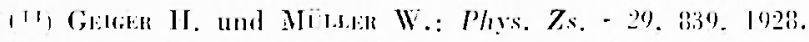

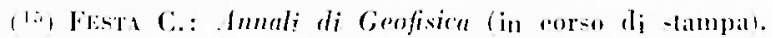

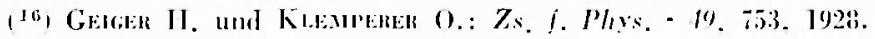

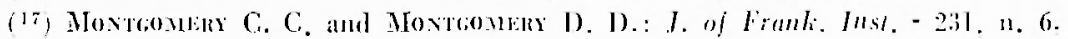
1911.

1151 Miz: R.: Journ. de Phys, 6. 61. 19.16.

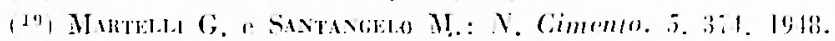

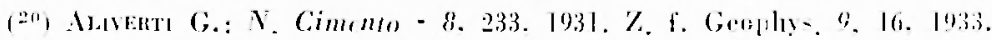

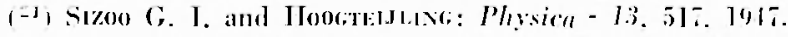

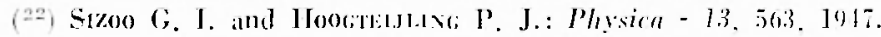

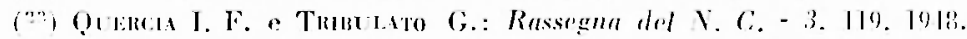

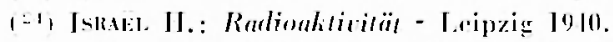

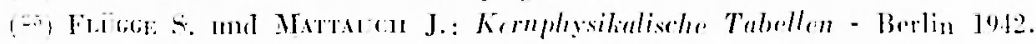

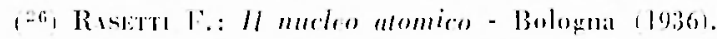

